\title{
ADAPTIVE FINITE ELEMENT SIMULATION OF INCOMPRESSIBLE FLOWS BY HYBRID CONTINUOUS-DISCONTINUOUS GALERKIN FORMULATIONS
}

\author{
SANTIAGO BADIA ${ }^{\dagger \ddagger ~ A N D ~ J O A N ~ B A I G E S ~} \ddagger \S$
}

\begin{abstract}
In this work we design hybrid continuous-discontinuous finite element spaces that permit discontinuities on non-matching element interfaces of non-conforming meshes. Then, we develop an equal-order stabilized finite element formulation for incompressible flows over these hybrid spaces, which combines the element interior stabilization of SUPGtype continuous Galerkin formulations and the jump stabilization of discontinuous Galerkin formulations. Optimal stability and convergence results are obtained. For the adaptive setting, we use an standard error estimator and marking strategy. Numerical experiments show the optimal accuracy of the hybrid algorithm both for uniformly and adaptively refined non-conforming meshes. The outcome of this work is a finite element formulation that can naturally be used on nonconforming meshes, as discontinuous Galerkin formulations, while keeping the much lower CPU cost of continuous Galerkin formulations.
\end{abstract}

Key words. Incompressible flows, adaptive refinement, continuous-discontinuous Galerkin, equal-order interpolation, stabilization

AMS subject classifications. 65N30, 35Q61, 65N12

1. Introduction. The dynamics of incompressible flows are governed by the incompressible Navier-Stokes equations, a set of nonlinear partial differential equations with a dissipative structure. Its numerical approximation is a challenging task. In the asymptotic regime of increasing Reynolds numbers the flow becomes chaotic (turbulent); mathematically, this is a singular limit with a coercivity loss [21]. On the other hand the system has a saddle-point (indefinite) structure, i.e. pressure stability relies on an inf-sup condition [37. Galerkin finite element (FE) approximations that satisfy a discrete inf-sup condition can solve the second issue but the coercivity loss constrains one to capture all the spatial scales of the flow, i.e. to reduce the computational mesh size up to the Kolmogorov microscale, which is unaffordable in many realistic applications due to limited computational resources.

Both issues can be solved by using residual-based FE stabilization techniques of SUPG-type 25. 26. 17, 19. These formulations make use of continuous FE spaces of functions, denoted as cG (continuous Galerkin) formulations onwards. The idea of these methods is to add to the Galerkin terms additional stabilizing terms that depend on the residual on the element interiors, keeping accuracy whereas improving stability. Stability does not depend on a discrete inf-sup condition anymore, and equal-order approximations can be used. Even for the Stokes problem, where the convective term does not appear, equal-order interpolation is very appealing in terms of implementation issues (simplicity of data-bases and matrix graphs) but also more efficient than inf-sup stable counterparts 31 .

Another approach to this problem is the use of non-conforming methods based on nodal discontinuous Galerkin $(\mathrm{dG})$ techniques [16, 22, 34. Convection stabilization is attained via a proper definition of the numerical fluxes [15, 38. Again, pressure stability can rely on a discrete inf-sup condition 34; the discrete inf-sup condition holds for $P_{k} / P_{k-1}$ and $Q_{k} / Q_{k-1}$ velocity-pressure pairs, with $k \geq 1$. On the other hand, equal order interpolations require additional stabilization terms that penalize pressure jumps [16, 15]. Cockburn and co-workers have concluded in [16] that equal-order dG formulations are more efficient.

Conforming cG formulations are preferred over $\mathrm{dG}$ formulations when we focus on CPU cost (at the same convergence order). For simplicial meshes, dG formulations involve around 14 times more degrees of freedom than $\mathrm{cG}$ ones in dimension three (6 in two dimensions) 1 For hexahedral meshes this ratio is around 8 and 4 for quadrilateral meshes. Certainly, these numbers cannot be ignored when simulating complex and realistic phenomena. However, the solution of many problems of interest often exhibit sharp

\footnotetext{
${ }^{\dagger}$ Centre Internacional de Mètodes Numèrics a l'Enginyeria (CIMNE), Parc Mediterrani de la Tecnologia, UPC, Esteve Terradas 5, 08860 Castelldefels, Spain (sbadia@cimne.upc.edu). The work of the first author was funded by the European Research Council under the FP7 Programme Ideas through the Starting Grant No. 258443 - COMFUS: Computational Methods for Fusion Technology.

${ }^{\ddagger}$ Universitat Politècnica de Catalunya, Jordi Girona 1-3, Edifici C1, 08034 Barcelona, Spain.

$\S$ Centre Internacional de Mètodes Numèrics a l'Enginyeria (CIMNE), Building C1, Campus Nord UPC, Gran Capità S/N 08034 Barcelona, Spain (jbaiges@cimne.upc.edu).

${ }^{1}$ Those are the values obtained for a structured mesh with periodic boundary conditions.
} 
layers or strong singularities. The use of locally refined meshes in these regions is required in order to get good results, since uniformly refined meshes can be prohibitive. dG formulations are better suited to adaptive refinement, because they can easily deal with non-conforming meshes with hanging nodes 27, e.g. using local mesh refinement, compared to $\mathrm{cG}$ formulations. We refer to 1 for the red-green mesh refinement strategy for $\mathrm{cG}$ formulations, which keeps the conformity of the mesh but not the aspect ratio. Alternatively, non-conforming refined meshes can be used together with cG formulations, by constraining the hanging nodes in order to keep continuity. This approach is certainly involved in terms of implementation and it is usually restricted to 1-irregular meshes [33, i.e. two neighboring elements can only differ in at most one level of refinement. The most general case in the $h p$-adaptivity setting has been faced in $[36$.

The motivation of this work is a hybrid method that combines the low CPU cost of cG formulations with the capabilities of $\mathrm{dG}$ formulations when dealing with adaptive refinement, naturally denoted as continuous-discontinuous Galerkin (cdG) formulation. In particular, we design an equal-order cdG numerical method for the approximation of incompressible flows, due to the superior efficiency and simplicity both in the $\mathrm{cG}$ and $\mathrm{dG}$ case. The cdG formulation is designed in such a way that the method is stable and optimally convergent for this particular type of FE spaces. The resulting methods is a suitable combination of the cG variational multiscale (VMS) formulation in [26] and an equal-order symmetric interior penalty $\mathrm{dG}$ formulation [15] with upwind for the convective term [13, 38.

The outline of the paper is as follows. In Section 2 we introduce the refined (non-conforming) meshes and hybrid cdG FE spaces. We design a hybrid cdG FE formulation for the Stokes problem in Section 3 . The well-posedness and optimal convergence of this formulation is proved in Section 4 . In Section 5 the method is extended to Oseen and Navier-Stokes systems, by introducing the convective term. Section 6 is devoted to the adaptive refinement strategy. A set of numerical experiments is included in Section 7. both for the steady Stokes and transient Navier-Stokes problems. Finally, some conclusions are drawn in Section 8

\section{Meshes and FE spaces.}

2.1. Refined meshes. Let us consider an open, bounded, Lipschitz polyhedral domain $\Omega$ in $\mathbb{R}^{d}$, where $d=2,3$ is the space dimension. Let $\mathcal{T}_{h}^{0}$ be a conforming and shape-regular partition of $\bar{\Omega}$ into tetrahedra (triangles for $d=2$ ) or hexahedra (quadrilaterals for $d=2$ ) where every $K \in \mathcal{T}_{h}$ is the image of a reference element $\widehat{K}$ through a smooth bijective mapping $F_{K}$.

Now, let us consider $\mathcal{T}_{h}$ to be obtained from the conforming mesh $\mathcal{T}_{h}^{0}$ via a finite number of refinement/coarsening steps:

$$
\left\{\mathcal{T}_{h}^{0}, \mathcal{T}_{h}^{1}, \ldots, \mathcal{T}_{h}^{m} \equiv \mathcal{T}_{h}\right\}, \quad \text { such that } \quad\left\{\mathcal{T}_{h}^{i}=\operatorname{REF}\left(\mathcal{T}_{h}^{i-1}, \Theta^{i}\right), i=1, \ldots, m<\infty\right\},
$$

where REF applies the refinement procedure over a given mesh $\mathcal{T}_{h}^{i-1}$ and $\Theta^{i}: \mathcal{T}_{h}^{i-1} \rightarrow\{-1,0,1\}$ is an array that establishes the action to be taken at each element: -1 for coarsening, 0 for "do nothing" and 1 for refinement. We refer to Section 6 for the computation of $\Theta$ and implementation aspects. 2

We consider a local refinement of an element $K$ that preserves the aspect ratio, i.e. every tetrahedron is sub-divided into 6 affine tetrahedra (4 for triangles); analogously for hexahedra. This way, we have a correspondence between the father element $K$ and the set of children elements, denoted by $\operatorname{chl}(K)$, generated by the refinement. Analogously, for every element $K$, we define its father as fat $(K)$; fat $(K)=\emptyset$ for $K \in \mathcal{T}_{h}^{0}$ and $\operatorname{chl}(K)=\emptyset$ for $K \in \mathcal{T}_{h}$. The set of sibling elements for an element $K$ is defined as $\operatorname{sib}(K):=\operatorname{chl}($ fat $(K))$. Clearly, $\mathcal{T}_{h}$ can be expressed in terms of $\mathcal{T}_{h}^{0}$ and ordered directed trees (tree data structures), one for every element $K \in \mathcal{T}_{h}^{0}$. We assume that $\Theta^{i}$ is defined in such a way that $\Theta^{i}(K)=-1$ only if $K \notin \mathcal{T}_{h}^{0}$ and $\Theta^{i}\left(K^{\prime}\right)=-1$ for every $K^{\prime} \in \operatorname{sib}(K)$. Further, we define the level of an element as follows:

$$
\operatorname{lev}(K)=0, \quad \text { for any } K \in \mathcal{T}_{h}^{0}, \quad \operatorname{lev}(K)=\operatorname{lev}(\operatorname{fat}(K))+1 \quad \text { otherwise. }
$$

See Fig.2.1 for a simple mesh refinement example and the associated tree structure.

\footnotetext{
${ }^{2}$ The convergence analysis in Section 4 applies for any $\mathcal{T}_{h}$ obtained via this refinement procedure, but is independent of the refinement steps; the coarsening process is not needed for this static case. In Section 6 where adaptivity is considered, we will make use of this sequence of triangulations.
} 

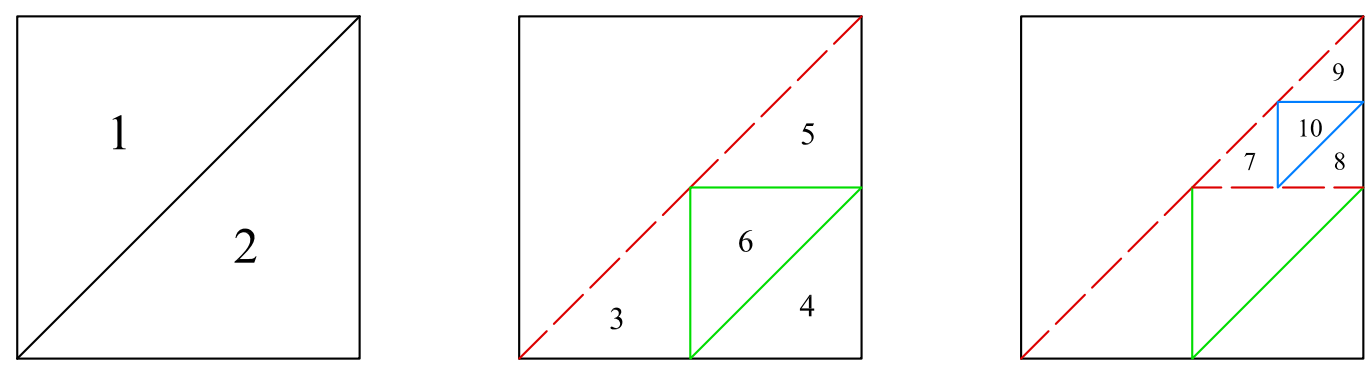

(1)

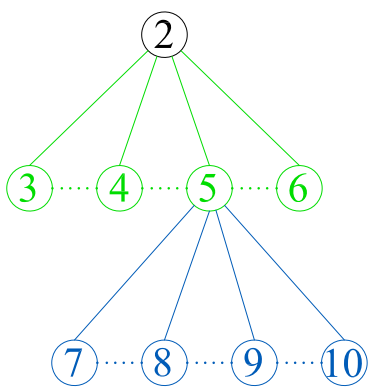

FIG. 2.1. Adaptive refinement mesh and its associated tree structure. Dashed lines represent improper faces.

Let $h_{K}$ denote the diameter of $K \in \mathcal{T}_{h}$ and $h:=\max _{K \in \mathcal{T}_{h}} h_{K}$. We assume a shape-regular partition: there exists a constant $\sigma \in(1, \infty)$ independent of $h$ such that $\max _{K \in \mathcal{T}_{h}} \frac{h_{K}}{\rho_{K}} \leq \sigma$, where $\rho_{K}$ denotes the radius of the largest closed ball contained in $K$. Let us note that $\sigma$ is the same for any mesh in the sequence (2.1), due to the affine refinement.

The refinement strategy proposed above is very simple compared to red-green refinement 11, specially in three dimensions, and keeps the aspect ratio $\sigma$ of the original triangulation $\mathcal{T}_{h}^{0}$. However, the resulting mesh is not conforming anymore, i.e. hanging nodes appear in the local refinement process.

We denote two elements $K$ and $K^{\prime}$ to be neighbors when meas $d_{-1}\left(\partial K \cap \partial K^{\prime}\right)>0$. We can easily check that the intersection between two neighboring elements can only be a common face or the entire face of one of the two elements 3 In the subsequent work, we allow for irregular meshes, but we assume that there exists a constant $\kappa>0$ independent of $h$ such that

$$
\kappa h_{K} \leq h_{K^{\prime}} \leq \kappa^{-1} h_{K}, \quad \text { for any pair of neighboring elements } K, K^{\prime} \in \mathcal{T}_{h} .
$$

In fact, $\kappa$ linearly depends on $\frac{1}{2 \llbracket \text { lev }}$ where $\llbracket$ lev $\rrbracket$ is the maximum difference between the level of refinement of two neighboring elements. So, we assume that $\llbracket$ lev $\rrbracket$ is bounded.

We denote by $\mathcal{N}_{h}(K):=\left\{\boldsymbol{x}_{K}^{(j)}, j=1, \ldots, n_{\text {nd }}\right\}$ the Lagrange nodes of each $K \in \mathcal{T}_{h}$, where $n_{\text {nd }}$ depends on the order of approximation of the FE space. The global set of Lagrange nodes is $\mathcal{N}_{h}:=$ $\bigcup_{K \in \mathcal{T}_{h}} \mathcal{N}_{h}(K)$. For every node $a \in \mathcal{N}_{h}$, we denote by $\omega_{a}:=\left\{K \in \mathcal{T}_{h}: a \in K\right\}$. Since $\mathcal{T}_{h}$ is shape-regular, $\operatorname{card}\left(\omega_{a}\right)$ is bounded, uniformly in $h$. Finally, let us denote by $\widetilde{\omega}_{a}:=\left\{K \in \omega_{a}: a \notin \mathcal{N}_{h}(K)\right\}$. Now, we can distinguish between proper nodes such that $\widetilde{\omega}_{a}=\emptyset$ and improper (or hanging) nodes (see also [27]).

Finally, let us define the concept of improper faces as follows. Given a pair of neighboring elements $K$ and $K^{\prime}$ such that lev $(K) \neq \operatorname{lev}\left(K^{\prime}\right), E:=\partial K \cap \partial K^{\prime}$ is an improper face (see Fig.2.1). We denote by $\mathcal{E}_{h}$ the union of improper faces in $\mathcal{T}_{h}$. Analogously to $h_{K}$, we can define $h_{E}$ as the diameter of $E \in \mathcal{E}_{h}$. However, it makes more sense to consider $h_{E}=\operatorname{dist}\left(\boldsymbol{b}_{K}, \boldsymbol{b}_{K^{\prime}}\right)$, where $\boldsymbol{b}_{K}$ are the barycentric coordinates of element $K$ (see [6]). For shape-regular partitions, the relation $\kappa^{\prime} h_{K} \leq h_{E} \leq \kappa^{\prime} h_{K}$ for some constant $\kappa^{\prime}$ independent of $h$ easily holds from (2.2).

2.2. Hybrid continuous-discontinuous FE spaces. Let us introduce some basic notation. Let $(q, \mathbf{v}, \boldsymbol{\tau})$ be scalar-, vector- and tensor-valued smooth functions inside every element $K \in \mathcal{T}_{h}$. Let $K^{+}$and

\footnotetext{
${ }^{3}$ We use generically "face" also for two-dimensional problems, in the place of "edge."
} 
$K^{-}$be two neighboring elements such that $\operatorname{lev}\left(K^{+}\right) \neq \operatorname{lev}\left(K^{-}\right)$and $E=\partial K^{+} \cap \partial K^{-}$the corresponding improper face. We denote by $\left(q^{ \pm}, \mathbf{v}^{ \pm}, \boldsymbol{\tau}^{ \pm}\right)$the traces of $(q, \mathbf{v}, \boldsymbol{\tau})$ on $\partial K^{ \pm}$taken within the interior of $K^{ \pm}$, and $\mathbf{n}^{ \pm}$the outward normal to $\partial K^{ \pm}$. We define at $\boldsymbol{x} \in E$ the averages

$$
\left\{\{q\}:=\frac{1}{2}\left(q^{+}+q^{-}\right), \quad\left\{\{\mathbf{v}\}:=\frac{1}{2}\left(\mathbf{v}^{+}+\mathbf{v}^{-}\right), \quad\left\{\{\boldsymbol{\tau}\}:=\frac{1}{2}\left(\boldsymbol{\tau}^{+}+\boldsymbol{\tau}^{-}\right),\right.\right.\right.
$$

and jumps

$$
\llbracket q \rrbracket:=\left(q^{+} \mathbf{n}^{+}+q^{-} \mathbf{n}^{-}\right), \quad \llbracket \mathbf{v} \rrbracket \quad:=\left(\mathbf{v}^{+} \cdot \mathbf{n}^{+}+\mathbf{v}^{-} \cdot \mathbf{n}^{-}\right), \quad \llbracket \boldsymbol{\tau} \rrbracket:=\left(\boldsymbol{\tau}^{+} \mathbf{n}^{+}+\boldsymbol{\tau}^{-} \mathbf{n}^{-}\right) .
$$

Further, we define $\llbracket \mathbf{v} \rrbracket:=\left(\mathbf{v}^{+} \otimes \mathbf{n}^{+}+\mathbf{v}^{-} \otimes \mathbf{n}^{-}\right)$.

Given an interpolation order of approximation $r$, we can denote the discontinuous FE space of functions as:

$$
X_{h}^{\mathrm{dG}}:=\left\{v_{h}:\left.v_{h}\right|_{K} \circ F_{K} \in P_{r}(\widehat{K}), K \in \mathcal{T}_{h}\right\} .
$$

$P_{r}(\widehat{K})$ is the space of complete polynomials of degree $r$ on $\widehat{K}$; alternatively, we can use the space of polynomials $Q_{r}(\widehat{K})$ with maximum degree $r$ in each reference space coordinate on $\widehat{K}$. For each $K \in \mathcal{T}_{h}$, the set of local basis functions corresponding to $\mathcal{N}_{h}(K)$ is denoted by $\left\{\phi_{K}^{(a)}, a \in \mathcal{N}_{h}(K)\right\}$, satisfying $\phi_{K}^{(a)}\left(\boldsymbol{x}^{(b)}\right)=$ $\delta_{a b}$. For dG approximations, the number of degrees of freedom (for an scalar quantity) coincides with $\sum_{K \in \mathcal{T}_{h}} \operatorname{card}\left(\mathcal{N}_{h}(K)\right)$; any function $v_{h} \in X_{h}^{\mathrm{dG}}$ can be written as $v_{h}=\sum_{K \in \mathcal{T}_{h}} \sum_{a \in \mathcal{N}_{h}(K)} v_{K}^{(a)} \phi_{K}^{(a)}$.

A continuous FE space is simply defined by $X_{h}^{\mathrm{cG}}:=X_{h}^{\mathrm{dG}} \cap C^{0}(\Omega)$. The definition of hybrid continuous-discontinuous spaces is more involved. For every node $a \in \mathcal{N}_{h}$, we define its corresponding shape function as $\phi^{(a)}:=\sum_{K \in \omega_{a} \backslash \widetilde{\omega}_{a}} \phi_{K}^{(a)}$. The cdG FE space is $X_{h}^{\text {cdG }}:=\operatorname{span}\left\{\phi^{(a)}: a \in \mathcal{N}_{h}\right\}$; clearly $\operatorname{dim}\left(X_{h}^{\text {cdG }}\right)=\operatorname{card}\left(\mathcal{N}_{h}\right)$. For conforming meshes, the cdG and cG FE spaces coincide. On the other hand, the cdG FE space includes degrees of freedom on hanging nodes whereas they must be eliminated from the cG space via the continuity enforcement (see 36] for practical implementations of these constraints). It implies a continuity loss on the set of improper faces $\mathcal{E}_{h}$ but does not require the introduction of complex (and possibly unsolvable) constraints [36. We may allow additional discontinuity over some FE interfaces, e.g. when a material discontinuity appears. We refer to 5 for the localized use of dG methods on material interfaces and the Maxwell problem. In addition, boundary conditions can be enforced weakly [30, 7]. In the subsequent work, we only allow jumps on improper faces due to the appearance of hanging nodes. The extension to additional discontinuous interfaces and weak boundary conditions is straightforward.

In the following, we will use $Q_{h}:=X_{h}^{\text {cdG }}$ for the pressure FE space, $V_{h}:=\left\{\mathbf{v}_{h} \in\left(X_{h}^{\text {cdG }}\right)^{d}:\right.$ $\mathbf{v}_{h}=\mathbf{0}$ on $\left.\partial \Omega\right\}$ for the velocity FE space and the auxiliary space of tensor-valued FE functions $\Sigma_{h}:=$ $\left(X_{h}^{\mathrm{cdG}}\right)^{d \times d}$.

3. Stabilized cdG formulation. Let us consider a polyhedral bounded domain $\Omega \subset \mathbb{R}, d=2,3$ filled with a viscous, incompressible Newtonian fluid. We denote by $\mathbf{n}$ the outward normal to its boundary $\partial \Omega$. Let us assume that the fluid is governed by the steady-state Stokes system: given a body force $\mathbf{f} \in L^{2}(\Omega)^{d}$, find a velocity $\mathbf{u}(\boldsymbol{x}) \in H_{0}^{1}(\Omega)^{d}$ and pressure $p(\boldsymbol{x}) \in L_{0}^{2}(\Omega)$ (the space of functions in $L^{2}(\Omega$ ) with zero mean value) such that

$$
-\nu \Delta \mathbf{u}+\nabla p=\mathbf{f}, \quad \nabla \cdot \mathbf{u}=0 \quad \text { in } \Omega,
$$

where $\nu>0$ is the kinematic viscosity; homogeneous boundary conditions are considered for simplicity. The variational formulation of the steady Stokes problem reads as follows: find $(\mathbf{u}, p) \in H_{0}^{1}(\Omega)^{d} \times L_{0}^{2}(\Omega)$ such that

$$
\begin{cases}A(\mathbf{u}, \mathbf{v})+B(\mathbf{v}, p) & =F(\mathbf{v}) \\ B(\mathbf{u}, q) & =0\end{cases}
$$

for any $(\mathbf{v}, q) \in H_{0}^{1}(\Omega)^{d} \times L_{0}^{2}(\Omega)$, where

$$
A(\mathbf{u}, \mathbf{v})=\int_{\Omega} \nabla \mathbf{u} \cdot \nabla \mathbf{v} \mathrm{d} \boldsymbol{x}, \quad B(\mathbf{v}, p)=-\int_{\Omega} p \nabla \cdot \mathbf{v} \mathrm{d} \boldsymbol{x}, \quad F(\mathbf{v})=\int_{\Omega} \mathbf{f} \cdot \mathbf{v} \mathrm{d} \boldsymbol{x} .
$$


The well-posedness of this boundary value problem relies on the inf-sup condition: for any $p \in L_{0}^{2}(\Omega)$ there exists $\mathbf{v}_{p} \in H_{0}^{1}(\Omega)^{d}$ with unit norm such that $\beta\|p\| \leq\left(p, \nabla \cdot \mathbf{v}_{p}\right)$, for a fixed constant $\beta>0$. Unlike coercivity, inf-sup conditions are not inherited by conforming FE subspaces of functions. One alternative is to consider a crude Galerkin aproximation using inf-sup stable elements [12. The statement of the discretized system in this case is straightforward from (3.2), simply replacing the infinite dimensional spaces $H_{0}^{1}(\Omega)^{d}$ and $L_{0}^{2}(\Omega)$ by the corresponding cG spaces. Unfortunately, appealing choices for the velocity and pressure FE spaces (like equal-order nodal interpolation) cannot be used. In addition, when applied to the Navier-Stokes equations (see Sections 5 and 7), the resulting method is not stable in the dominant convection limit. Both problems, pressure and convection stability can be solved by using residual-based stabilized FE methods [25, 26, 17, 19. In this work, we consider a variational multiscale (VMS) formulation in the pure cG case [26]; a cdG formulation with symmetric stabilization [18, 8, 29, 3] could be considered instead.

For dG FE spaces, an equal-order formulation for the (Navier)-Stokes and Oseen problems has been proposed in [16, 15]; we refer to these works for the statement of the full dG formulation. In particular, we consider the symmetric interior penalty (IP) formulation, since this dG formulation is stated in terms of $\left(\mathbf{u}_{h}, p_{h}\right)$ only and so, it can be combined with the cG formulation.

We combine the VMS cG solver [26] and the IP dG solver in [15] in such a way that the resulting method is stable for the cdG case. We anticipate that both formulations are unstable when the cdG space is used. The cG formulation assumes continuity of functions and no jump stabilization is introduced. The dG method assumes some inclusions between FE spaces which are not true in the cdG case, e.g. $\nabla Q_{h} \not \subset V_{h}$ [16. The motivation of the jump terms in the cdG formulation is standard of dG formulations 2. 13. Further, we have used the fact that the cdG FE functions can only be discontinuous on improper faces. Now, the cdG formulation reads as: find $\left(\mathbf{u}_{h}, p_{h}\right) \in V_{h} \times Q_{h}$ such that

$$
\begin{cases}A_{h}\left(\mathbf{u}_{h}, \mathbf{v}_{h}\right)+B_{h}\left(\mathbf{v}_{h}, p_{h}\right) & =F_{h}\left(\mathbf{v}_{h}\right) \\ -B_{h}\left(\mathbf{u}_{h}, q_{h}\right)+S_{h}\left(p_{h}, q_{h}\right) & =G_{h}\left(q_{h}\right)\end{cases}
$$

for any $\left(\mathbf{v}_{h}, q_{h}\right) \in V_{h} \times Q_{h}$, with the bilinear forms

$$
\begin{aligned}
A_{h}\left(\mathbf{u}_{h}, \mathbf{v}_{h}\right)= & \int_{\Omega} \nu \nabla_{h} \mathbf{u}_{h}: \nabla_{h} \mathbf{v}_{h} \mathrm{~d} \boldsymbol{x}-\int_{\mathcal{E}_{h}}\left\{\nu \nabla_{h} \mathbf{u}_{h}\right\}: \underline{\llbracket \mathbf{v}_{h} \rrbracket} \mathrm{d} \sigma-\int_{\mathcal{E}_{h}}\left\{L \nabla_{h} \mathbf{v}_{h}\right\}: \llbracket \underline{\llbracket \mathbf{u}_{h} \rrbracket} \mathrm{d} \sigma \\
& +\int_{\mathcal{E}_{h}} \delta_{\mathrm{ju}} \underline{\llbracket \mathbf{u}_{h} \rrbracket}: \underline{\llbracket \mathbf{v}_{h} \rrbracket} \mathrm{d} \sigma-\int_{\Omega} \delta_{\text {inu }} \nu^{2} \Delta_{h} \mathbf{u}_{h} \cdot \Delta_{h} \mathbf{v}_{h} \mathrm{~d} \boldsymbol{x}, \\
B_{h}\left(\mathbf{v}_{h}, q_{h}\right)= & -\int_{\Omega} q_{h} \nabla_{h} \cdot \mathbf{v}_{h} \mathrm{~d} \boldsymbol{x}+\int_{\mathcal{E}_{h}}\left\{q_{h}\right\} \llbracket \mathbf{v}_{h} \rrbracket \mathrm{d} \sigma-\int_{\Omega} \delta_{\text {inu }} \nu \nabla_{h} q_{h} \cdot \Delta_{h} \mathbf{v}_{h} \mathrm{~d} \boldsymbol{x}, \\
S_{h}\left(p_{h}, q_{h}\right)= & \int_{\Omega} \delta_{\text {inu }} \nabla_{h} p_{h} \cdot \nabla_{h} q_{h} \mathrm{~d} \boldsymbol{x}+\int_{\mathcal{E}_{h}} \delta_{\mathrm{jp}} \llbracket p_{h} \rrbracket \cdot \llbracket q_{h} \rrbracket \mathrm{d} \sigma,
\end{aligned}
$$

and linear forms

$$
F_{h}\left(\mathbf{v}_{h}\right)=\int_{\Omega} \mathbf{f}_{h} \cdot \mathbf{v}_{h} \mathrm{~d} \boldsymbol{x}+\int_{\Omega} \delta_{\text {inu }} \nu \mathbf{f}_{h} \cdot \Delta_{h} \mathbf{v}_{h} \mathrm{~d} \boldsymbol{x}, \quad G_{h}\left(q_{h}\right)=\int_{\Omega} \delta_{\text {inu }} \mathbf{f}_{h} \cdot \nabla_{h} q_{h} \mathrm{~d} \boldsymbol{x} .
$$

The values of the stabilization parameters are:

$$
\delta_{\mathrm{inu}}(\boldsymbol{x}):=\frac{c_{\mathrm{inu}} h_{K}^{2}}{\nu} \quad \text { for } \boldsymbol{x} \in K, \quad \delta_{\mathrm{ju}}(\boldsymbol{x}):=\frac{c_{\mathrm{ju}} \nu}{h_{E}} \quad \text { for } \boldsymbol{x} \in E, \quad \delta_{\mathrm{jp}}(\boldsymbol{x}):=\frac{c_{\mathrm{jp}} h_{E}}{\nu} \quad \text { for } \boldsymbol{x} \in E,
$$

where $c_{\mathrm{inu}}, c_{\mathrm{ju}}$ and $c_{\mathrm{jp}}$ are algorithmic constants; $c_{\mathrm{inu}}$ and $c_{\mathrm{ju}}$ are assumed to satisfy (4.8) below.

For cG FE spaces the VMS formulation [26] is obtained by eliminating the jump terms. We have not included the so-called grad-div term, since it is not essential for the following analysis [17. On the other hand, for dG FE spaces the IP equal-order dG formulation in [15] is obtained by taking $\delta_{\text {inu }}=0$ and including all the element boundaries in $\mathcal{E}_{h}$. The resulting formulation (3.3) combines the $\mathrm{cG}$ and $\mathrm{dG}$ formulations in such a way that the cdG formulation is stable and optimally convergent (see Theorems 4.2 and 4.4 below). On the one hand, we include an interior element stabilization of VMS type that allows us to violate the inclusion $\nabla Q_{h} \subset V_{h}$. On the other hand, dG jump terms are kept on the improper 
faces only. As a result, we have a numerical formulation that permits to reduce the number of degrees of freedom to the $\mathrm{cG}$ case but inheriting the capabilities of $\mathrm{dG}$ approaches to handle non-conforming meshes.

4. Numerical analysis. In this section, we analyze the stability and convergence properties of the numerical method (3.3). We will make use of the local inverse estimates for FE functions (see [11])

$$
\left\|\nabla_{h} v_{h}\right\|_{K} \leq c_{I} h_{K}^{-1}\left\|v_{h}\right\|_{K}, \quad\left\|\Delta_{h} v_{h}\right\|_{K} \leq c_{I} h_{K}^{-1}\left\|\nabla v_{h}\right\|_{K}, \quad \text { for any } K \in \mathcal{T}_{h} .
$$

In addition, we have the following trace inequality:

$$
\|v\|_{\partial K}^{2} \leq c_{T}\left(h_{K}^{-1}\|v\|_{K}^{2}+h_{K}\|\nabla v\|_{K}^{2}\right), \quad \text { for any } v \in H^{1}(K), K \in \mathcal{T}_{h} .
$$

For FE functions, combining these two inequalities, we get $\left\|v_{h}\right\|_{\partial K}^{2} \leq c_{T}\left(1+c_{I}^{2}\right) h_{K}^{-1}\left\|v_{h}\right\|_{K}^{2}$. Further, let us assume that the FE spaces to be used have optimal interpolation properties. We assume the existence of a linear operator $i_{h}(\cdot)$ such that, for any $v \in W_{2}^{k}(\Omega)$ and $0 \leq s \leq k \leq r+1, i_{h}(v) \in X_{h}^{\text {cdG }}$ satisfies

$$
\left\|v-i_{h}(v)\right\|_{W_{2}^{s}(K)} \leq c_{e} h_{K}^{k-s}\|v\|_{W_{2}^{k}\left(S_{K}\right)}, \quad\left(\sum_{K \in \mathcal{T}_{h}}\left\|v-i_{h}(v)\right\|_{W_{2}^{s}(K)}^{2}\right)^{\frac{1}{2}} \leq c_{e} h^{k-s}\|v\|_{W_{2}^{k}(\Omega)} .
$$

$c_{I}, c_{T}$ and $c_{e}$ are positive fixed constants independent of $h . S_{K}$ is a domain made of the neighboring elements of $K$ and $r$ denotes the order of approximation of the corresponding FE space. The global estimate only holds for shape-regular partitions. In the dG limit, the local estimate in fact applies for $S_{K}=K$ [16]. We refer to [35] for a proof of [4.3). The approach in [35] can be extended to cdG FE spaces, defining the node-to-element mapping in 35] in such a way that a node $a$ can only be associated to a $K \in \omega_{a} \backslash \widetilde{\omega}_{a}$.

For the subsequent analysis, it is convenient to rewrite (3.4a)- (3.4b) by using lifting operators 2 . Let $\mathcal{L}_{h}: V_{h}+H_{0}^{1}(\Omega)^{d} \rightarrow \Sigma_{h}$ such that

$$
\int_{\Omega} \mathcal{L}_{h}(\mathbf{v}): \boldsymbol{\tau}_{h} \mathrm{~d} \boldsymbol{x}=\int_{\mathcal{E}_{h}}\left\{\left\{\boldsymbol{\tau}_{h}\right\}: \underline{\llbracket \mathbf{v} \rrbracket} \mathrm{d} \sigma, \quad \text { for any } \boldsymbol{\tau}_{h} \in \Sigma_{h},\right.
$$

as well as $\mathcal{M}_{h}: V_{h}+H_{0}^{1}(\Omega)^{d} \rightarrow Q_{h}$ such that

$$
\int_{\Omega} q_{h} \mathcal{M}_{h}(\mathbf{v}) \mathrm{d} \boldsymbol{x}=\int_{\mathcal{E}_{h}}\left\{\left\{q_{h}\right\} \llbracket \llbracket \mathbf{v} \rrbracket \mathrm{d} \sigma, \quad \text { for any } q_{h} \in Q_{h} .\right.
$$

The following stability results apply for these operators:

$$
\left\|\mathcal{L}_{h}(\mathbf{v})\right\|^{2} \leq c_{L} \sum_{E \in \mathcal{E}_{h}} h_{E}^{-1}\|\underline{\llbracket} \rrbracket\|_{E}^{2}, \quad\left\|\mathcal{M}_{h}(\mathbf{v})\right\|^{2} \leq c_{L} \sum_{E \in \mathcal{E}_{h}} h_{E}^{-1}\|\llbracket \mathbf{v} \rrbracket\|_{E}^{2},
$$

for some constant $c_{L}>0$ independent of $h$. We omit the proof since it follows the same lines as for the $\mathrm{dG}$ case in 32. Let us consider the modified bilinear forms

$$
\begin{aligned}
A_{h}(\mathbf{u}, \mathbf{v})= & \int_{\Omega} \nu \nabla_{h} \mathbf{u}: \nabla_{h} \mathbf{v} \mathrm{d} \boldsymbol{x}-\int_{\Omega} \mathcal{L}_{h}(\mathbf{v}): \nu \nabla_{h} \mathbf{u} \mathrm{d} \boldsymbol{x}-\int_{\Omega} \mathcal{L}_{h}(\mathbf{u}): \nu \nabla_{h} \mathbf{v} \mathrm{d} \boldsymbol{x} \\
& +\int_{\mathcal{E}_{h}} \delta_{\mathrm{ju}} \underline{\llbracket \mathbf{u} \rrbracket}: \underline{\underline{\mathbb{v}} \rrbracket} \mathrm{d} \sigma-\int_{\Omega} \delta_{\mathrm{inu}} \nu^{2} \Delta_{h} \mathbf{u} \cdot \Delta_{h} \mathbf{v} \mathrm{d} \boldsymbol{x}, \\
B_{h}(\mathbf{v}, q)= & -\int_{\Omega} q \nabla_{h} \cdot \mathbf{v} \mathrm{d} \boldsymbol{x}+\int_{\Omega} q \mathcal{M}_{h}(\mathbf{v}) \mathrm{d} \boldsymbol{x}-\int_{\Omega} \delta_{\text {inu }} \nu \nabla_{h} q \cdot \Delta_{h} \mathbf{v} \mathrm{d} \boldsymbol{x},
\end{aligned}
$$

which are obtained by using the lifting operators (4.4)-(4.5) in place of their respective terms in (3.4a)(3.4b). We make abuse of notation, denoting these forms the same way as those in (3.4a)-(3.4b), since the numerical method (3.3) does not change with these modifications, i.e., the bilinear forms (4.7a)-4.7b) are identical to (3.4a)-(3.4b) when restricted to FE functions in $V_{h} \times Q_{h}$. In contrast to (3.4a)-(3.4b), 
(4.7a)-(4.7b) are also well-defined for velocity functions in $H_{0}^{1}(\Omega)^{d}$ and pressure functions in $L_{0}^{2}(\Omega)$. This re-statement of the problem is only needed for numerical analysis purposes.

Further, we assume that

$$
c_{\mathrm{inu}}<\frac{1-\varsigma}{c_{I}^{2}}, \quad c_{\mathrm{ju}}>\frac{c_{L}}{\varsigma}, \quad \text { for some } \varsigma>0 .
$$

Clearly, this is always possible, i.e. take $\varsigma=\frac{1}{2}$. Roughly speaking, $c_{\text {inu }}$ is assumed to be small enough and $c_{\mathrm{ju}}$ large enough. For the $\mathrm{cG}$ VMS method and the dG IP methods, this is always required (see e.g. [4] and [2], respectively).

Finally, the symbol $\lesssim$ is used to denote $\leq$ up to a positive constant independent of $h$ (idem for $\gtrsim$ ).

LEMma 4.1. The bilinear forms in (3.4) satisfy the following inequalities.

(i) Coercivity of $A_{h}$ : For any $\mathbf{u}_{h} \in V_{h}$,

$$
A_{h}\left(\mathbf{u}_{h}, \mathbf{u}_{h}\right) \gtrsim \nu\left\|\nabla_{h} \mathbf{u}_{h}\right\|^{2}+\int_{\mathcal{E}_{h}} \delta_{\mathrm{ju}}\left|\underline{\llbracket \mathbf{u}_{h} \rrbracket}\right|^{2} \mathrm{~d} \sigma
$$

(ii) Continuity of $A_{h}$ : For any $\mathbf{u}, \mathbf{v} \in V_{h}+H^{2}(\Omega)^{d} \cap H_{0}^{1}(\Omega)^{d}$,

$$
\begin{aligned}
A_{h}(\mathbf{u}, \mathbf{v}) \lesssim(\nu \| & \left.\nabla_{h} \mathbf{u} \|^{2}+\int_{\mathcal{E}_{h}} \delta_{\mathrm{ju}} \underline{\left.\underline{\llbracket \mathbf{u} \rrbracket}\right|^{2}} \mathrm{~d} \sigma+\int_{\Omega} \delta_{\text {inu }} \nu^{2}\left|\Delta_{h} \mathbf{u}\right|^{2} \mathrm{~d} \boldsymbol{x}\right)^{\frac{1}{2}} \\
& \times\left(\nu\left\|\nabla_{h} \mathbf{v}\right\|^{2}+\int_{\mathcal{E}_{h}} \delta_{\mathrm{ju}} \underline{|\underline{\mathbb{v} \rrbracket}|^{2}} \mathrm{~d} \sigma+\int_{\Omega} \delta_{\text {inu }} \nu^{2}\left|\Delta_{h} \mathbf{v}\right|^{2} \mathrm{~d} \boldsymbol{x}\right)^{\frac{1}{2}} .
\end{aligned}
$$

(iii) Weak coercivity of $S_{h}:$ For any $p_{h} \in Q_{h}$

$$
S_{h}\left(p_{h}, p_{h}\right) \geq \int_{\Omega} \delta_{\text {inu }}\left|\nabla_{h} p_{h}\right|^{2} \mathrm{~d} \boldsymbol{x}+\int_{\mathcal{E}_{h}} \delta_{\mathrm{jp}}\left|\llbracket p_{h} \rrbracket\right|^{2} \mathrm{~d} \sigma
$$

(iv) Continuity of $S_{h}$ : For any $p, q \in Q_{h}+H^{1}(\Omega) \cap L_{0}^{2}(\Omega)$

$$
S_{h}(p, q) \lesssim\left(\int_{\Omega} \delta_{\text {inu }}\left|\nabla_{h} p\right|^{2} \mathrm{~d} \boldsymbol{x}+\int_{\mathcal{E}_{h}} \delta_{\mathrm{jp}}|\llbracket p \rrbracket|^{2} \mathrm{~d} \sigma\right)^{\frac{1}{2}} \times\left(\int_{\Omega} \delta_{\text {inu }}\left|\nabla_{h} q\right|^{2} \mathrm{~d} \boldsymbol{x}+\int_{\mathcal{E}_{h}} \delta_{\mathrm{jp}}|\llbracket q \rrbracket|^{2} \mathrm{~d} \sigma\right)^{\frac{1}{2}} .
$$

(v) Continuity of $B_{h}$ : For any $\mathbf{v} \in V_{h}+H^{2}(\Omega)^{d} \cap H_{0}^{1}(\Omega)^{d}$ and $q \in Q_{h}+H^{1}(\Omega) \cap L_{0}^{2}(\Omega)$

$$
\begin{aligned}
B_{h}(\mathbf{v}, q) \lesssim\left(\nu^{-1}\|q\|^{2}+\int_{\Omega} \delta_{\text {inu }}\left|\nabla_{h} q\right|^{2} \mathrm{~d} \boldsymbol{x}\right)^{\frac{1}{2}} & \\
& \times\left(\nu\left\|\nabla_{h} \cdot \mathbf{v}\right\|^{2}+\left.\int_{\mathcal{E}_{h}} \delta_{\text {ju }} \underline{\llbracket \mathbf{v} \rrbracket}\right|^{2} \mathrm{~d} \sigma+\int_{\Omega} \delta_{\text {inu }} \nu^{2}\left|\Delta_{h} \mathbf{v}\right|^{2} \mathrm{~d} \boldsymbol{x}\right)^{\frac{1}{2}} .
\end{aligned}
$$

Proof. In order to get the first result, we bound the jump terms that do not provide stability by using the stability properties of the lifting operators and Young's inequality:

$$
2 \int_{\Omega} \nu \mathcal{L}_{h}\left(\mathbf{u}_{h}\right): \nabla_{h} \mathbf{u}_{h} \mathrm{~d} \boldsymbol{x} \leq \varsigma \nu\left\|\nabla_{h} \mathbf{u}_{h}\right\|^{2}+\frac{c_{L}}{\varsigma c_{\mathrm{ju}}} \int_{\mathcal{E}_{h}} \delta_{\mathrm{ju}} \underline{\left.\underline{\llbracket \mathbf{u}_{h} \rrbracket}\right|^{2}} \mathrm{~d} \sigma
$$

for an arbitrary constant $\varsigma>0$. The interior stabilization term can also be bounded by using the second inverse inequality in (4.1):

$$
\int_{\Omega} \delta_{\text {inu }} \nu^{2}\left|\Delta_{h} \mathbf{u}_{h}\right|^{2} \mathrm{~d} \boldsymbol{x} \leq c_{I}^{2} \sum_{K \in \mathcal{T}_{h}} \frac{\delta_{\text {inu }} \nu^{2}}{h_{K}^{2}} \int_{K}\left|\nabla_{h} \mathbf{u}_{h}\right|^{2} \mathrm{~d} \boldsymbol{x} \leq c_{I}^{2} c_{\text {inu }} \nu\left\|\nabla_{h} \mathbf{u}_{h}\right\|^{2} .
$$

Combining these results with the assumption (4.8) over the constants $c_{\mathrm{inu}}$ and $c_{\mathrm{ju}}$, we prove (4.9). The continuity result (4.10) is obtained using similar arguments. 
The third result is straightforward and the fourth one follows by using Cauchy-Schwarz inequality. Finally, the continuity of $B_{h}$ is obtained as follows. The jump term is bounded by using the continuity of the lifting operators in (4.6) and the definition of $\delta_{\mathrm{ju}}$ :

$$
\int_{\Omega} q \mathcal{M}_{h}(\mathbf{v}) \mathrm{d} \boldsymbol{x} \leq\|q\|\left\|\mathcal{M}_{h}(\mathbf{v})\right\| \leq \nu^{-\frac{1}{2}}\|q\|\left(\frac{c_{L}}{c_{\mathrm{ju}}} \int_{\mathcal{E}_{h}} \delta_{\mathrm{ju}} \|\left.\mathbf{v} \rrbracket\right|^{2} \mathrm{~d} \sigma\right)^{\frac{1}{2}} .
$$

The interior terms are readily bounded by the Cauchy-Schwarz inequality.

With these results, we can prove the following discrete (weak) inf-sup condition.

ThEOREM 4.2. There exists a positive constant $\beta_{p}$ such that, for each $p_{h} \in Q_{h}$ :

$$
\beta_{p} \nu^{-\frac{1}{2}}\left\|p_{h}\right\| \leq \sup _{\mathbf{v}_{h} \in V_{h}} \frac{B_{h}\left(\mathbf{v}_{h}, p_{h}\right)}{\left(\nu\left\|\nabla_{h} \mathbf{v}_{h}\right\|^{2}+\int_{\mathcal{E}_{h}}\left|\llbracket \mathbf{v}_{h} \rrbracket\right|^{2} \mathrm{~d} \sigma\right)^{\frac{1}{2}}}+S_{h}\left(p_{h}, p_{h}\right)^{\frac{1}{2}}
$$

Proof. Due to the continuous inf-sup condition [37, for any $p_{h} \in Q_{h} \subset Q$ there exists $\mathbf{v}_{p} \in H_{0}^{1}(\Omega)^{d}$ such that $\nu^{-1}\left\|p_{h}\right\|^{2} \lesssim-\left(p_{h}, \nabla \cdot \mathbf{v}_{p}\right)$ and $\nu^{\frac{1}{2}}\left\|\mathbf{v}_{p}\right\|_{1}=\nu^{-\frac{1}{2}}\left\|p_{h}\right\|$. This is equivalent to say that $\nu^{-1}\left\|p_{h}\right\|^{2} \lesssim$ $\left.B\left(\mathbf{v}_{p}, p_{h}\right)+\int_{\mathcal{E}_{h}}\left\{p_{h}\right\}\right\} \llbracket \mathbf{v}_{p} \rrbracket \mathrm{d} \sigma$, since $\llbracket \mathbf{v}_{p} \rrbracket=0$. Next, let us consider the projection $i_{h} \mathbf{v}_{p}:=i_{h}\left(\mathbf{v}_{p}\right) \in V_{h}$ with optimal interpolation properties. Using the following equality, obtained by integration-by-parts,

$$
-\int_{\Omega} q_{h} \nabla_{h} \cdot \mathbf{v} \mathrm{d} \boldsymbol{x}+\int_{\mathcal{E}_{h}}\left\{\left\{q_{h}\right\} \rrbracket \llbracket \mathbf{v} \rrbracket \mathrm{d} \sigma=\int_{\Omega}\left(\nabla_{h} q_{h}\right) \cdot \mathbf{v} \mathrm{d} \boldsymbol{x}-\int_{\mathcal{E}_{h}} \llbracket q_{h} \rrbracket \cdot\{\{\mathbf{v}\} \mathrm{d} \sigma,\right.
$$

which holds for any $q_{h} \in Q_{h}$ and $\mathbf{v} \in V_{h}+H_{0}^{1}(\Omega)$, we have:

$$
\begin{aligned}
\nu^{-1}\left\|p_{h}\right\|^{2} \lesssim-\int_{\Omega} p_{h} \nabla_{h} \cdot \mathbf{v}_{p} \mathrm{~d} \boldsymbol{x}+ & \int_{\mathcal{E}_{h}}\left\{p_{h}\right\} \llbracket \llbracket \mathbf{v}_{p} \rrbracket \mathrm{d} \sigma=\int_{\Omega}\left(\nabla_{h} p_{h}\right) \cdot\left(\mathbf{v}_{p}-i_{h} \mathbf{v}_{p}\right) \mathrm{d} \boldsymbol{x} \\
& \left.-\int_{\mathcal{E}_{h}} \llbracket p_{h} \rrbracket \cdot\left\{\mathbf{v}_{p}-i_{h} \mathbf{v}_{p}\right\}\right\} \mathrm{d} \sigma+B_{h}\left(i_{h} \mathbf{v}_{p}, p_{h}\right)+\int_{\Omega} \delta_{\text {inu }} \nu \nabla_{h} p_{h} \cdot \Delta i_{h} \mathbf{v}_{p} \mathrm{~d} \boldsymbol{x} .
\end{aligned}
$$

The first two terms in the right-hand side of the previous inequality can be bounded as follows:

$$
\begin{aligned}
\int_{\Omega}\left(\nabla_{h} p_{h}\right) & \cdot\left(\mathbf{v}_{p}-i_{h} \mathbf{v}_{p}\right) \mathrm{d} \boldsymbol{x}-\int_{\mathcal{E}_{h}} \llbracket p_{h} \rrbracket \cdot\left\{\left\{\mathbf{v}_{p}-i_{h} \mathbf{v}_{p}\right\} \mathrm{d} \sigma\right. \\
& \lesssim\left(\int_{\Omega} \delta_{\text {inu }}\left|\nabla_{h} p_{h}\right|^{2} \mathrm{~d} \boldsymbol{x}+\int_{\mathcal{E}_{h}} \delta_{\text {jp }}\left|\llbracket p_{h} \rrbracket\right|^{2} \mathrm{~d} \sigma\right)^{\frac{1}{2}} \nu^{-\frac{1}{2}}\left\|p_{h}\right\| .
\end{aligned}
$$

where we have used the trace inequality (4.2), the interpolation properties in (4.3), the definition of the stabilization parameters (3.6) and the stability of the projector, i.e. $\left\|i_{h} \mathbf{v}\right\|_{1} \lesssim\|\mathbf{v}\|_{1}$ (consequence of (4.3)). Further, using the second inverse inequality (4.1) and the fact that $\nu^{\frac{1}{2}}\left\|\mathbf{v}_{p}\right\|_{1}=\nu^{-\frac{1}{2}}\left\|p_{h}\right\|$, we get

$$
-\int_{\Omega} \delta_{\text {inu }} \nu \nabla_{h} p_{h} \cdot \Delta_{h} i_{h} \mathbf{v}_{p} \mathrm{~d} \boldsymbol{x} \lesssim\left(\int_{\Omega} \delta_{\text {inu }}\left|\nabla_{h} p_{h}\right|^{2} \mathrm{~d} \boldsymbol{x}\right)^{\frac{1}{2}} \nu^{\frac{1}{2}}\left\|\nabla_{h} i_{h} \mathbf{v}_{p}\right\| \lesssim\left(\int_{\Omega} \delta_{\text {inu }}\left|\nabla_{h} p_{h}\right|^{2} \mathrm{~d} \boldsymbol{x}\right)^{\frac{1}{2}} \nu^{-\frac{1}{2}}\left\|p_{h}\right\| .
$$

Invoking (4.17), (4.18) and (4.9) in (4.16), and dividing the resulting inequality by $\nu^{-\frac{1}{2}}\left\|p_{h}\right\|$, we end up with the following bound:

$$
\nu^{-\frac{1}{2}}\left\|p_{h}\right\| \lesssim \frac{B_{h}\left(i_{h} \mathbf{v}_{p}, p_{h}\right)}{\nu^{-\frac{1}{2}}\left\|p_{h}\right\|}+S_{h}\left(p_{h}, p_{h}\right)^{\frac{1}{2}}
$$

Furthermore, using (4.1), (4.2) and (4.3) we obtain:

$$
\nu\left\|\nabla_{h} i_{h} \mathbf{v}_{p}\right\|^{2}+\int_{\mathcal{E}_{h}} \delta_{\mathrm{ju}}\left|\llbracket i_{h} \mathbf{v}_{p} \rrbracket\right|^{2} \mathrm{~d} \sigma \lesssim \nu\left\|\nabla_{h} \mathbf{v}_{p}\right\|^{2}+\int_{\mathcal{E}_{h}} \delta_{\mathrm{ju}}\left|\llbracket \mathbf{v}_{p}-i_{h} \mathbf{v}_{p} \rrbracket\right|^{2} \mathrm{~d} \sigma \leq \nu\left\|\nabla_{h} \mathbf{v}_{p}\right\|^{2} \lesssim \nu^{-1}\left\|p_{h}\right\|^{2} .
$$


Combining (4.19) and (4.20) we prove the theorem.

Let us define the global norm

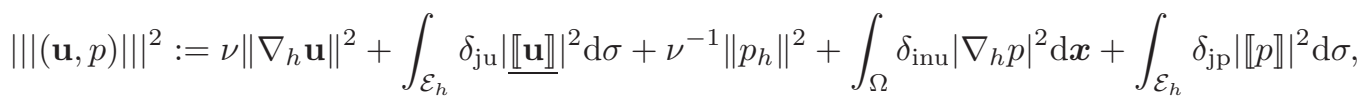

as well as the global bilinear form

$$
C_{h}((\mathbf{u}, p),(\mathbf{v}, q)):=A_{h}(\mathbf{u}, \mathbf{v})+B_{h}(\mathbf{v}, p)-B_{h}(\mathbf{u}, q)+S_{h}(p, q) .
$$

We have the following stability result.

COROLlary 4.3. There exists a positive constant $\beta$ such that:

$$
\inf _{\left(\mathbf{v}_{h}, q_{h}\right) \in V_{h} \times Q_{h}} \sup _{\left(\mathbf{w}_{h}, r_{h}\right) \in V_{h} \times Q_{h}} \frac{C_{h}\left(\left(\mathbf{v}_{h}, q_{h}\right),\left(\mathbf{w}_{h}, r_{h}\right)\right)}{\left\|\left(\mathbf{v}_{h}, q_{h}\right)\right\| \times\left|\left\|\left(\mathbf{w}_{h}, r_{h}\right)\right\|\right|} \geq \beta .
$$

Proof. Combining (4.9) and (4.11) we obtain the following bound:

$$
\begin{aligned}
& A_{h}\left(\mathbf{u}_{h}, \mathbf{u}_{h}\right)+B_{h}\left(\mathbf{u}_{h}, p_{h}\right)-B_{h}\left(\mathbf{u}_{h}, p_{h}\right)+S_{h}\left(p_{h}, p_{h}\right) \\
& \quad \gtrsim\left\|\nabla_{h} \mathbf{u}_{h}\right\|^{2}+\int_{\mathcal{E}_{h}} \delta_{\mathrm{ju}}\left|\llbracket \mathbf{u}_{h} \rrbracket\right|^{2} \mathrm{~d} \sigma+\int_{\Omega} \delta_{\text {inu }}\left|\nabla_{h} p_{h}\right|^{2} \mathrm{~d} \boldsymbol{x}+\int_{\mathcal{E}_{h}} \delta_{\mathrm{jp}}\left|\llbracket p_{h} \rrbracket\right|^{2} \mathrm{~d} \sigma .
\end{aligned}
$$

In addition, we know from Theorem 4.2 that there exists a $\mathrm{FE}$ function $\mathbf{v}_{p, h} \in V_{h}$ such that $\nu\left\|\nabla_{h} \mathbf{v}_{p, h}\right\|^{2}+$ $\left.\int_{\mathcal{E}_{h}} \delta_{\mathrm{ju}} \underline{\llbracket \mathbf{v}_{p, h} \rrbracket}\right|^{2} \mathrm{~d} \sigma=\nu^{-1}\left\|p_{h}\right\|^{2}$ and

$$
\frac{\beta_{p}}{2} \nu^{-1}\left\|p_{h}\right\|^{2}-\frac{1}{2 \beta_{p}}\left(\int_{\Omega} \delta_{\text {inu }}\left|\nabla_{h} p_{h}\right|^{2} \mathrm{~d} \boldsymbol{x}+\int_{\mathcal{E}_{h}} \delta_{\mathrm{jp}}\left|\llbracket p_{h} \rrbracket\right|^{2} \mathrm{~d} \sigma\right) \leq B_{h}\left(\mathbf{v}_{p, h}, p_{h}\right)
$$

Further, using the continuity of $A_{h}$ in (4.10) and the inequality (4.14), which holds for any $\mathbf{v}_{h} \in V_{h}$, we obtain:

$$
\begin{aligned}
& A_{h}\left(\mathbf{u}_{h}, \mathbf{v}_{p, h}\right)+B_{h}\left(\mathbf{v}_{p, h}, p_{h}\right) \gtrsim \beta_{p}\left\|p_{h}\right\|^{2}-\frac{1}{2 \beta_{p}}\left(\int_{\Omega} \delta_{\text {inu }}\left|\nabla_{h} p_{h}\right|^{2} \mathrm{~d} \boldsymbol{x}+\int_{\mathcal{E}_{h}} \delta_{\mathrm{jp}}\left|\llbracket p_{h} \rrbracket\right|^{2} \mathrm{~d} \sigma\right) \\
& -\frac{\varsigma}{2}\left(\nu\left\|\nabla_{h} \mathbf{u}_{h}\right\|^{2}+\int_{\mathcal{E}_{h}} \delta_{\mathrm{ju}}\left|\underline{\llbracket \mathbf{u}_{h} \rrbracket}\right|^{2} \mathrm{~d} \sigma\right)-\frac{1}{2 \varsigma}\left(\nu\left\|\nabla_{h} \mathbf{v}_{p, h}\right\|^{2}+\int_{\mathcal{E}_{h}} \delta_{\mathrm{ju}}\left|\underline{\llbracket \mathbf{v}_{p, h} \rrbracket}\right|^{2} \mathrm{~d} \sigma\right)
\end{aligned}
$$

Using the definition of $\mathbf{v}_{p, h}$ and taking $\varsigma$ large enough, we get

$$
\begin{aligned}
A_{h}\left(\mathbf{u}_{h}, \mathbf{v}_{p, h}\right)+B_{h}\left(\mathbf{v}_{p, h}, p_{h}\right) \gtrsim & || p_{h} \|^{2}-\int_{\Omega} \delta_{\text {inu }}\left|\nabla_{h} p_{h}\right|^{2} \mathrm{~d} \boldsymbol{x}-\int_{\mathcal{E}_{h}} \delta_{\mathrm{jp}}\left|\llbracket p_{h} \rrbracket\right|^{2} \mathrm{~d} \sigma \\
& -\left(\nu\left\|\nabla_{h} \mathbf{u}_{h}\right\|^{2}+\int_{\mathcal{E}_{h}} \delta_{\mathrm{ju}}\left|\underline{\llbracket \mathbf{u}_{h} \rrbracket}\right|^{2} \mathrm{~d} \sigma\right) .
\end{aligned}
$$

So, the inf-sup holds for $\left(\mathbf{v}_{h}, q_{h}\right)=\left(\mathbf{u}_{h}, p_{h}\right)+\alpha\left(\mathbf{v}_{p, h}, 0\right)$, with $\alpha$ small enough.

Finally, we obtain an a priori error estimate. It is a direct consequence of Lemma 4.1 and Corollary 4.3

THEOREM 4.4. Let the solution of the continuous problem (3.2) be such that $(\mathbf{u}, p) \in H^{1+\tau}(\Omega) \times$ $H^{\tau}(\Omega)$ for some $\tau \geq 1$, and $\left(\mathbf{u}_{h}, p_{h}\right)$ the solution of (3.3). The following error estimate holds:

$$
\left\|\left|\left(\mathbf{u}-\mathbf{u}_{h}, p-p_{h}\right)\right|\right\| \lesssim\left(\sum_{K \in \mathcal{T}_{h}} h_{K}^{2 k} \nu|\mathbf{u}|_{W_{2}^{k+1}\left(S_{K}\right)}^{2}+\sum_{K \in \mathcal{T}_{h}} h_{K}^{2 m} \nu^{-1}|p|_{W_{2}^{m}\left(S_{K}\right)}^{2}\right)^{\frac{1}{2}},
$$

with $k:=\min (\tau, r)$ and $m:=\min (\tau, r+1), r$ being the order of approximation of $V_{h}$ and $Q_{h}$.

Proof. Due to the consistency of (3.3) for $(\mathbf{u}, p) \in H^{1+\tau}(\Omega) \times H^{\tau}(\Omega)$, the Galerkin orthogonality holds:

$$
C_{h}\left(\left(\mathbf{u}-\mathbf{u}_{h}, p-p_{h}\right),\left(\mathbf{v}_{h}, q_{h}\right)\right)=0, \quad \text { for any }\left(\mathbf{v}_{h}, q_{h}\right) \in V_{h} \times Q_{h} .
$$


Thus, for an arbitrary pair $\left(\mathbf{w}_{h}, r_{h}\right) \in V_{h} \times Q_{h}$, we have:

$$
C_{h}\left(\left(\mathbf{w}_{h}-\mathbf{u}_{h}, r_{h}-p_{h}\right),\left(\mathbf{v}_{h}, q_{h}\right)\right)=C_{h}\left(\left(\mathbf{w}_{h}-\mathbf{u}, r_{h}-p\right),\left(\mathbf{v}_{h}, q_{h}\right)\right) .
$$

In this equality, invoking the stability result in Corollary 4.3, the continuity properties in (4.10), (4.12), (4.13) and the bound (4.15), we prove that there exists a $\left(\mathbf{v}_{h}, q_{h}\right) \in V_{h} \times Q_{h}$ such that:

$$
\begin{aligned}
\left\|\left|( \mathbf { w } _ { h } - \mathbf { u } _ { h } , r _ { h } - p _ { h } ) \left\||| \times||\left|\left(\mathbf{v}_{h}, q_{h}\right) \|\right|\right.\right.\right. & \lesssim C_{h}\left(\left(\mathbf{w}_{h}-\mathbf{u}_{h}, r_{h}-p_{h}\right),\left(\mathbf{v}_{h}, q_{h}\right)\right) \\
& \lesssim\left(\left\|\left.||\left(\mathbf{w}_{h}-\mathbf{u}, r_{h}-p\right)\left|\|^{2}+\int_{\Omega} \delta_{\text {inu }} \nu^{2}\right| \Delta_{h}\left(\mathbf{w}_{h}-\mathbf{u}\right)\right|^{2} \mathrm{~d} \boldsymbol{x}\right)^{\frac{1}{2}} \times\left\|\mid\left(\mathbf{v}_{h}, q_{h}\right)\right\| \| .\right.
\end{aligned}
$$

Using the triangle inequality and the previous inequality we get:

$$
\begin{aligned}
\left\|\left|\left(\mathbf{u}-\mathbf{u}_{h}, p-p_{h}\right)\right|\right\| \mid & \leq||\left|\left(\mathbf{w}_{h}-\mathbf{u}, r_{h}-p\right)\right|\left\||+|||\left(\mathbf{w}_{h}-\mathbf{u}_{h}, r_{h}-p_{h}\right)\right\| \mid \\
& \lesssim\left(\|\left.\left|\left(\mathbf{w}_{h}-\mathbf{u}, r_{h}-p\right)\right|\right|^{2}+\int_{\Omega} \delta_{\text {inu }} \nu^{2}\left|\Delta_{h}\left(\mathbf{w}_{h}-\mathbf{u}\right)\right|^{2} \mathrm{~d} \boldsymbol{x}\right)^{\frac{1}{2}} .
\end{aligned}
$$

Finally, picking $\left(\mathbf{w}_{h}, r_{h}\right)=\left(i_{h} \mathbf{u}, i_{h} p\right)$, and using the local interpolation error estimates (4.3) and the trace inequality (4.2), we easily get:

$$
\left\|\left|\left(\mathbf{w}_{h}-\mathbf{u}, r_{h}-p\right)\right|\right\|^{2}+\int_{\Omega} \delta_{\mathrm{inu}} \nu^{2}\left|\Delta_{h}\left(\mathbf{w}_{h}-\mathbf{u}\right)\right|^{2} \mathrm{~d} \boldsymbol{x} \lesssim \sum_{K \in \mathcal{T}_{h}} h_{K}^{2 k} \nu|\mathbf{u}|_{W_{2}^{k+1}\left(S_{K}\right)}^{2}+\sum_{K \in \mathcal{T}_{h}} h_{K}^{2 m} \nu^{-1}|p|_{W_{2}^{m}\left(S_{K}\right)}^{2}
$$

This proves the theorem.

5. Convective terms. In this section we extend the formulation (3.3) with the convective term, in order to consider the Oseen or Navier-Stokes problem. In the first case, the fluid is governed by the following system of equations: for any $t \in[0, T]$, given a body force $\mathbf{f}(\boldsymbol{x}, t) \in L^{2}(\Omega)^{d}$, find a velocity $\mathbf{u}(\boldsymbol{x}, t) \in H_{0}^{1}(\Omega)^{d}$ and pressure $p(\boldsymbol{x}, t) \in L_{0}^{2}(\Omega)$ such that

$$
\partial_{t} \mathbf{u}+(\mathbf{a} \cdot \nabla) \mathbf{u}-\nu \Delta \mathbf{u}+\nabla p=\mathbf{f}, \quad \nabla \cdot \mathbf{u}=0 \quad \text { in } \Omega \times[0, T]
$$

where $\mathbf{a} \in H_{0}^{1}(\Omega)^{d}$ is a divergence-free convective field. The nonlinear Navier-Stokes system is obtained by taking $\mathbf{a} \leftarrow \mathbf{u}$. The variational formulation of the steady Oseen problem reads as follows: find $(\mathbf{u}, p) \in H_{0}^{1}(\Omega)^{d} \times L_{0}^{2}(\Omega)$ such that

$$
\begin{cases}\left(\partial_{t} \mathbf{u}, \mathbf{v}\right)+A(\mathbf{u}, \mathbf{v})+D(\mathbf{a} ; \mathbf{u}, \mathbf{v})+B(\mathbf{v}, p) & =F(\mathbf{v}) \\ B(\mathbf{u}, q) & =0\end{cases}
$$

for any $(\mathbf{v}, q) \in H_{0}^{1}(\Omega)^{d} \times L_{0}^{2}(\Omega)$, almost everywhere in time. The convective term is written in skewsymmetric form for numerical purposes [37]:

$$
D(\mathbf{a} ; \mathbf{u}, \mathbf{v})=\int_{\Omega} \xi(\mathbf{a}, \mathbf{u}) \cdot \mathbf{v d} \boldsymbol{x}, \quad \text { with } \quad \xi(\mathbf{a}, \mathbf{u})=(\mathbf{a} \cdot \nabla) \mathbf{u}+\frac{1}{2}(\nabla \cdot \mathbf{a}) \mathbf{u} .
$$

Let us design a discretization of the convective term in the cdG formulation. It is obtained by using the original upwind formulation of the convective term (see e.g. [38]) in this case:

$$
\int_{K} \xi(\mathbf{a}, \mathbf{u}) \cdot \mathbf{v} \mathrm{d} \boldsymbol{x}+\int_{\partial K_{\mathrm{in}}} \mathbf{a} \cdot \mathbf{n}\left(\mathbf{u}^{e}-\mathbf{u}\right) \cdot \mathbf{v} \mathrm{d} \sigma
$$

We denote by $\partial K_{\text {in }}:=\left\{\boldsymbol{x} \in \partial K \cap \mathcal{E}_{h}: \mathbf{a} \cdot \mathbf{n}_{K}<0\right\}$, i.e. the inflow part of $\partial K \cap \mathcal{E}_{h}$. For every improper face $E \in \mathcal{E}_{h}$ such that $E \cap \partial K_{\text {in }} \neq \emptyset$, we denote by $K^{e}$ the neighboring element that shares $E$ and $\mathbf{u}^{e}$ is the external value of $\mathbf{u}_{h}$ on the edge, i.e. the value taken from inside $K^{e}$ [23, 38. This is the version that is better suited for the cdG case, since this is the usual way we treat the convective term in residualbased stabilized cG formulations, i.e. the non-conservative and skew-symmetric form. Additional ways to state this and related methods can be found in [13. On the other hand, the extension of the VMS 
formulation to the Oseen and Navier-Stokes systems is simple; the additional interior residual terms must be included. Unfortunately, the extension to transient problems of VMS formulations is not so straightforward. In this work, we have considered a so-called quasi-static approach, but more involved dynamic methods could be considered [17, 19. The treatment of the time derivative is transparent for symmetric stabilization techniques [18, 8, 29, 3. Let us consider the $\theta$-method for the time integration; we define $\dot{\mathbf{u}}^{n+1}:=\frac{1}{\delta t}\left(\mathbf{u}^{n+1}-\mathbf{u}^{n}\right)$ where $\delta t:=t^{n+1}-t^{n}$ is the time step size. Combining these concepts, we end up with Algorithm 1, where we have included the grad-div VMS stabilization.

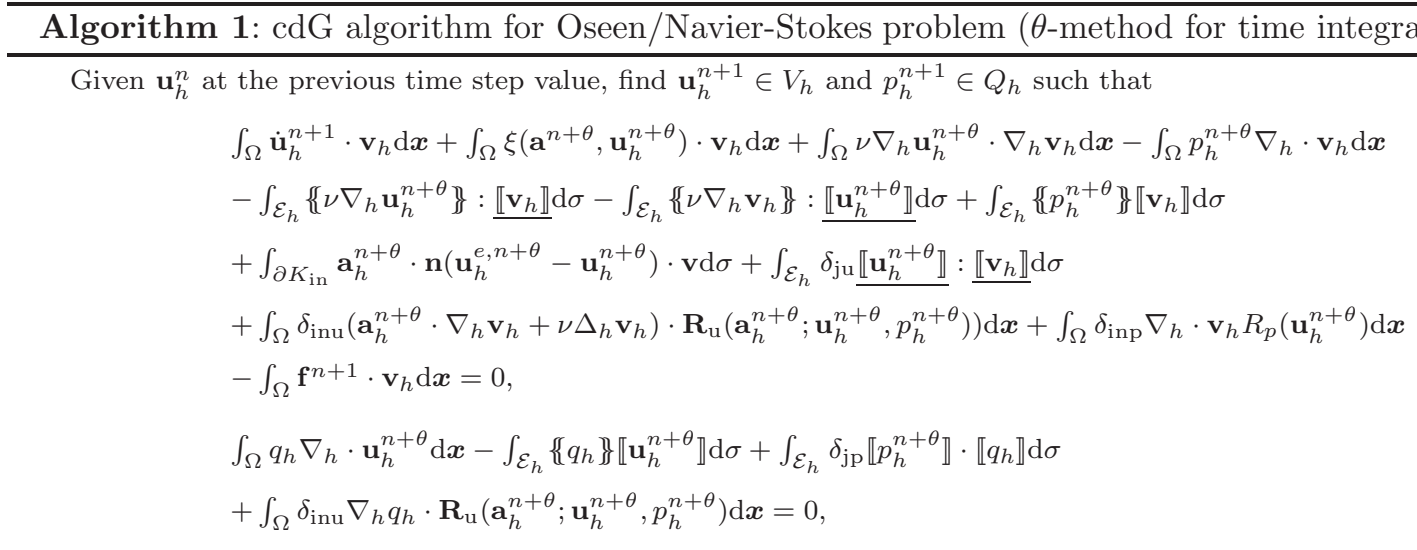

where the residuals are:

$$
\mathbf{R}_{\mathrm{u}}\left(\mathbf{a} ; \mathbf{u}_{h}, p_{h}\right):=\dot{\mathbf{u}}_{h}+\mathbf{a} \cdot \nabla_{h} \mathbf{u}_{h}-\nu \Delta_{h} \mathbf{u}_{h}+\nabla_{h} p_{h}-\mathbf{f}, \quad R_{p}\left(\mathbf{u}_{h}\right):=\nabla \cdot \mathbf{u}_{h} .
$$

The stabilization parameters have the following expressions within each element $K$ or edge $E$ :

$$
\begin{aligned}
\delta_{\mathrm{inu}}(\boldsymbol{x}):=\frac{c_{\mathrm{inu}} h_{K}^{2}}{\nu}+\frac{c_{\mathrm{inc}} h_{K}}{\|\mathbf{a}\| L^{\infty}(K)}, & \delta_{\mathrm{inp}}:=\frac{c_{\mathrm{inp}} h_{K}^{2}}{\delta_{\mathrm{inu}}} \quad \text { for } \boldsymbol{x} \in K, \\
\delta_{\mathrm{ju}}(\boldsymbol{x}):=\frac{c_{\mathrm{ju}} \nu}{h_{E}}, & \delta_{\mathrm{jp}}(\boldsymbol{x}):=\frac{c_{\mathrm{jp}} h_{E}}{\nu} \quad \text { for } \boldsymbol{x} \in E,
\end{aligned}
$$

where $c_{\mathrm{inu}}, c_{\mathrm{inc}}, c_{\mathrm{inp}}, c_{\mathrm{ju}}$ and $c_{\mathrm{jp}}$ are algorithmic constants that satisfy 4.8.

\section{Adaptive refinement strategy.}

6.1. Error indicators. A standard error indicator for the Stokes system can be found in [24]. For each $K \in \mathcal{T}_{h}$, we define the following error indicator $\eta_{K}$ as follows:

$$
\begin{aligned}
\eta_{K}^{2}:= & \alpha_{1} \nu^{-1} h_{K}^{2} \int_{K}\left|\mathbf{f}+\nu \Delta_{h} \mathbf{u}_{h}-\nabla_{h} p_{h}\right|^{2} \mathrm{~d} \boldsymbol{x}+\alpha_{2} \nu \int_{K}\left|\nabla_{h} \cdot \mathbf{u}_{h}\right|^{2} \mathrm{~d} \boldsymbol{x} \\
& +\alpha_{3} \nu^{-1} h_{K} \int_{\partial K \backslash \Gamma}\left|\llbracket \boldsymbol{\sigma}_{h} \rrbracket\right|^{2} \mathrm{~d} \sigma+\left.\alpha_{4} \nu h_{K}^{-1} \int_{\partial K} \underline{\mid \llbracket \mathbf{u}_{h} \rrbracket}\right|^{2} \mathrm{~d} \sigma .
\end{aligned}
$$

where $\boldsymbol{\sigma}_{h}:=p_{h} \boldsymbol{I}-\nu \nabla_{h} \mathbf{u}_{h}, \boldsymbol{I}$ is the identity tensor, and $\alpha_{i}$ are constants independent of $h$. The efficiency and reliability of the indicator has been proved for different velocity-pressure spaces [22, 23]. In the continuous Galerkin case, this indicator has also been used in [10, 9]; obviously, the last term is identically zero in this case. For the hybrid cdG case, the last term will only be positive on improper faces.

Following [28, the stress jump boundary terms can be replaced by interior element terms by using the Oswald quasi-interpolant $\pi_{h}^{*}$. This is possible because the following inequalities hold (see 14, Corollary 3.2] and [28]):

$$
\gamma_{1} h_{K} \sum_{K \in \mathcal{T}_{h}} \int_{\partial K \backslash \Gamma}\left|\llbracket \boldsymbol{\sigma}_{h} \rrbracket\right|^{2} \mathrm{~d} \sigma \leq\left\|\boldsymbol{\sigma}_{h}-\pi_{h}^{*}\left(\boldsymbol{\sigma}_{h}\right)\right\|^{2} \leq \gamma_{2} h_{K} \sum_{K \in \mathcal{T}_{h}} \int_{\partial K \backslash \Gamma}\left|\llbracket \boldsymbol{\sigma}_{h} \rrbracket\right|^{2} \mathrm{~d} \sigma
$$

where $\gamma_{1}$ and $\gamma_{2}$ are constants independent of $h$. Roughly speaking, we are replacing this boundary terms by a Zienkiewicz-Zhu estimator [39, 40] over stresses. This allows us to avoid computing edge 
integrals over proper faces. The error indicator now reads:

$$
\begin{aligned}
\eta_{K}^{2}:= & \alpha_{1} \nu^{-1} h_{K}^{2} \int_{K}\left|\mathbf{f}+\nu \Delta_{h} \mathbf{u}_{h}-\nabla_{h} p_{h}\right|^{2} \mathrm{~d} \boldsymbol{x}+\alpha_{2} \nu \int_{K}\left|\nabla_{h} \cdot \mathbf{u}_{h}\right|^{2} \mathrm{~d} \boldsymbol{x} \\
& +\alpha_{3} \nu^{-1}\left\|\boldsymbol{\sigma}_{h}-\pi_{h}^{*}\left(\boldsymbol{\sigma}_{h}\right)\right\|^{2}+\left.\alpha_{4} \nu h_{K}^{-1} \int_{\partial K} \underline{\mid \llbracket \mathbf{u}_{h} \rrbracket}\right|^{2} \mathrm{~d} \sigma .
\end{aligned}
$$

6.2. Marking criteria. A key ingredient for the correct performance of the adaptive refinement strategy is the election of the elements which need to be refined/coarsened. As explained in the previous sections, this selection is going to be based on the error estimator 6.3). Two basic strategies can be adopted for the adaptive refinement:

- Define an objective local error per element, and proceed with the refinement procedure until the local error of all elements is lower than the objective local error.

- Define an objective number of elements, proceed with the refinement procedure until the number of elements approaches the objective and the local error is approximately uniform in all the elements.

Both approaches lead to a mesh configuration where the error distribution is approximately uniform. Particularly, the second option involves the need of coarsening the elements in which the error is smaller while at the same time the elements with the largest local error are refined. In order to achieve this we rely on a tree-type structure in which we store the information regarding the ancestors and descendants of each element (see Section 2). Storing the information corresponding to this tree-structure allows us to easily perform the refinement/coarsening steps, as well as easily identifying the improper FE faces where the $\mathrm{dG}$ terms need to be integrated.

6.2.1. Marking criterion for the error objective strategy. In the case an objective local error per element is seeked, the refinement/coarsening criterion is defined in Algorithm 2, where $e_{\text {est }}$

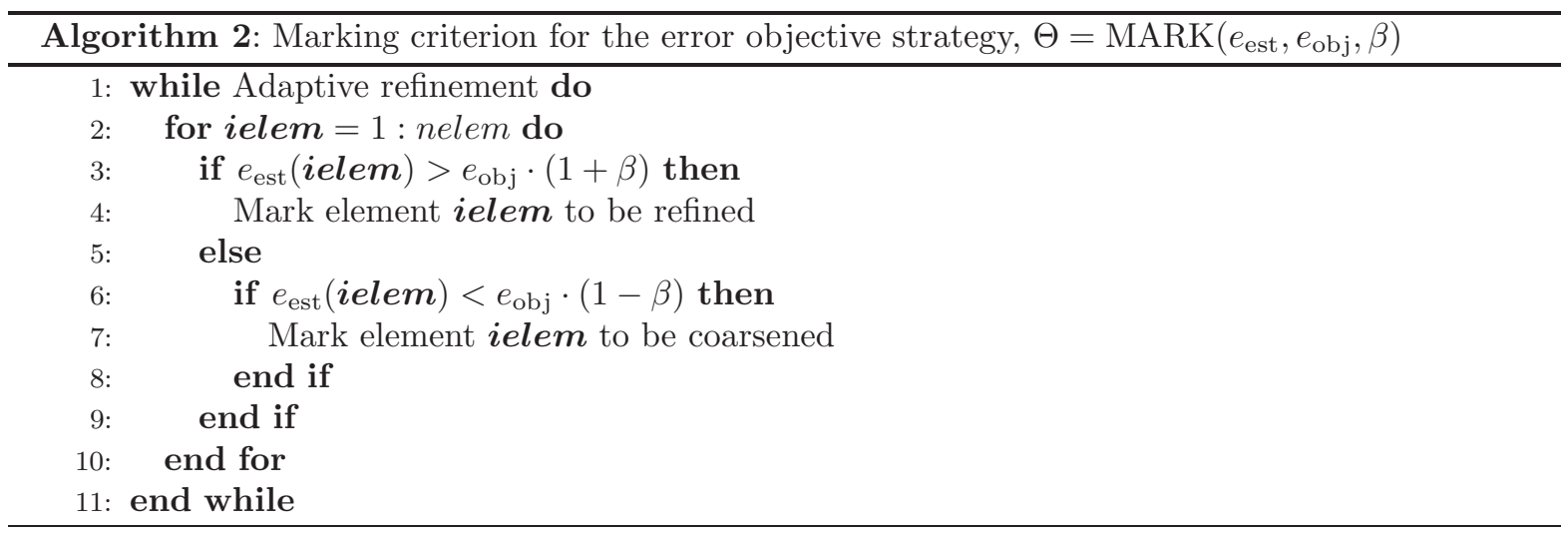

is the estimated local elemental error, $e_{\mathrm{obj}}$ is the objective elemental error and $\beta$ is a parameter which ranges from 0 to 1 , and represents a tolerance of the refinement criterion. Note that when an element is marked to be coarsened in Algorithm 2, the coarsening step will only be performed if all of its sibling elements are also marked as elements to be coarsened. As a result of this algorithm, we get the array $\Theta$ used for the mesh refinement in Section 2 .

6.2.2. Marking criterion for the number of elements objective strategy. When an objective number of elements is seeked, the refinement/coarsening criterion is defined in Algorithm 3. Again, $\beta$ plays the role of a parameter which adjusts the performance of the algorithm, affecting the speed at which the algorithm adapts to the objective number of elements. For the numerical examples in Section 7. $\beta=12$ was employed. Again, the output of this algorithm is the array $\Theta$.

Algorithm 2 and Algorithm 3 iteratively refine the FE mesh. In stationary problems this adaptive process is stopped when a stationary state is reached, that is, when the algorithm does no longer change the adaptive refinement mesh. On the other hand, the adaptive procedure runs continuously in the case of dynamical problems, where the large error regions can move through the physical domain during the 


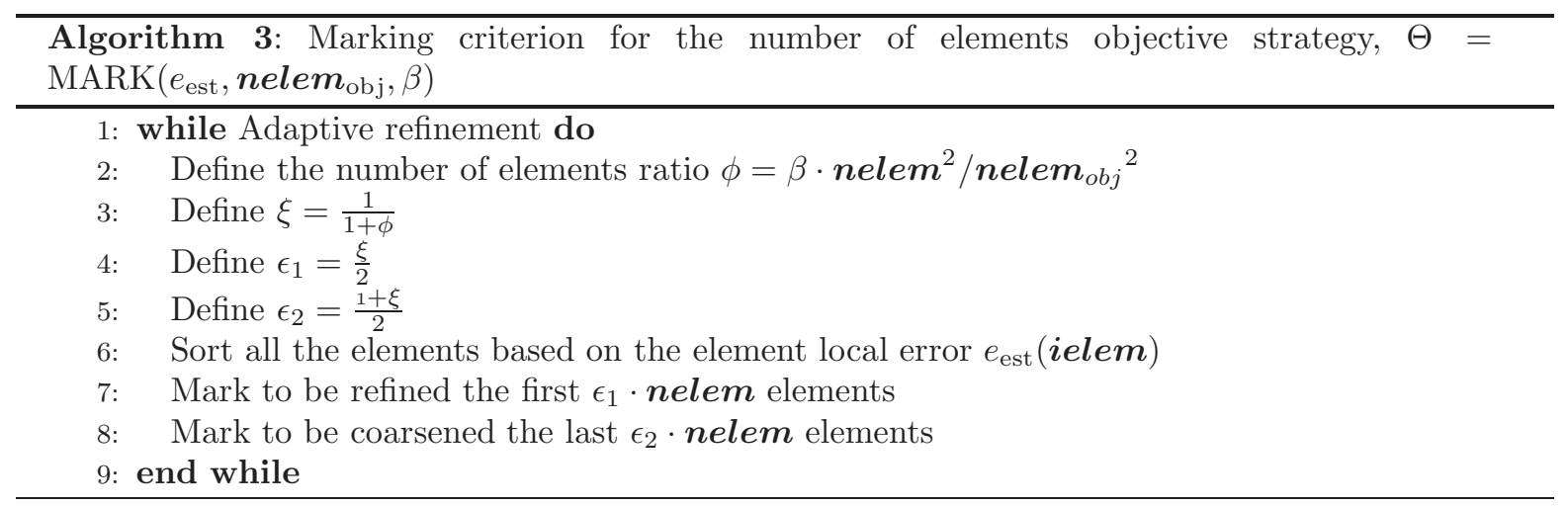

computation. This is illustrated in the last exemple of Section 7, where the incompressible flow around a cylinder is simulated. The vortexes which appear at the wake of the cylinder make the refined FE mesh evolve in time, following the sharp velocity gradients.

After each refinement step, some care needs to be taken in the imposition of boundary conditions in the new mesh. When an analytical solution is available, the new boundary nodes are imposed the exact value for the field of interest. In general cases, given a new node on a refined boundary edge, we only fix a velocity component when it has been fixed on the two nodes of the parent edge. The value is interpolated with the FE basis, in order to preserve the original boundary conditions. We proceed identically for new nodes on the interior of parent faces for three-dimensional problems. The value of the previous time step velocity on new nodes is also interpolated for dynamical problems.

\section{Numerical examples.}

7.1. Smooth solution problem. In this numerical example we test the performance of the cdG formulation (3.3) and the proposed adaptive refinement strategy with a smooth analytical solution for the Stokes problem:

$$
\mathbf{u}(x, y)=\left[\begin{array}{c}
2 x y \\
-y^{2}
\end{array}\right], \quad p(x, y)=x y-\frac{1}{4}, \quad \text { on } \quad \Omega:=(0,1) \times(0,1) .
$$

Fig.7.1 shows the velocity and pressure fields obtained from the numerical simulations. For the adaptive refinement strategy, we start from a structured mesh of 8 linear triangular FEs and we let Algorithm 2 refine the mesh until the error estimator at each element reaches a certain cutoff value. Due to the smoothness of the solution, the resulting adaptive FE meshes are very close to the ones obtained if a uniform refinement strategy is used. Fig.7.2 shows one of the adaptive meshes after several refinement steps. It can be observed that, for a certain element error threshold, at most two element sizes appear. In fact, for certain error thresholds the mesh resulting from the adaptive refinement strategy coincides exactly with the corresponding uniform refinement mesh. This can be seen in Fig.7.3, where the convergence plots for the adaptive velocity and pressure fields overlap with the convergence plots of the structured uniform meshes at certain points. When the uniform and adaptive meshes do not coincide, uniform refinement overperforms adaptive refinement for this smooth solution problem. This is due to the fact that the global error is governed by the largest local element error, which corresponds to the error of the large elements in the adaptive mesh (Fig.7.2). The convergence order is optimal for the velocity field $\left(\mathcal{O}\left(h^{2}\right) \sim \mathcal{O}(1 / n)\right)$ and superconvergent for the pressure field $(\mathcal{O}(h) \sim \mathcal{O}(\sqrt{1 / n})$, where $h$ denotes the element size and $n$ the number of mesh nodes. It is also interesting to observe the performance of the error estimator in the adaptive refinement procedure. Fig.7.4 shows the estimated error evaluated as detailed in (6.3) versus the exact error measured in the cdG energy norm (4.21) as well as the effectivity index, i.e. the ratio between the estimated and exact error. It can be observed that the convergence rate is the same for the error estimator and the exact error, as well as the excellent behavior of the error estimator with an efectivity index around 1. Fig.7.5 shows the ratio between $h_{K} \sum_{K \in \mathcal{T}_{h}} \int_{\partial K \backslash \Gamma}\left|\llbracket \boldsymbol{\sigma}_{h} \rrbracket\right|^{2} \mathrm{~d} \boldsymbol{x}$ and $\left\|\boldsymbol{\sigma}_{h}-\pi_{h}^{*}\left(\boldsymbol{\sigma}_{h}\right)\right\|^{2}$ which is the base for the error estimator (6.3). The figure illustrates the equivalence between both terms, which converge at the same rate, the boundary 
terms being approximately two times larger than the interior element terms. A comparison between the number of unknowns of the proposed cdG formulation and a pure dG formulation for the same FE mesh is presented in Fig.77.6. The ratio between the number of unknowns is around 6 for this bidimensional problem.
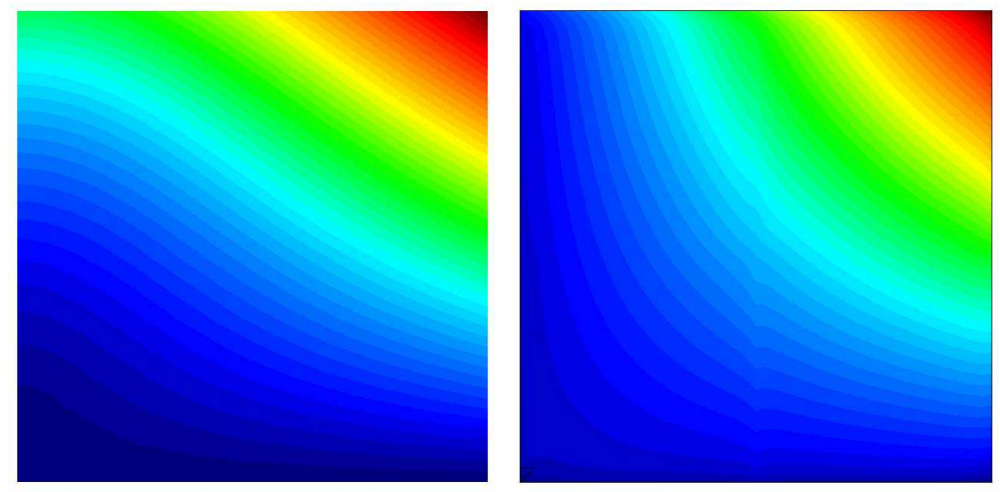

FIG. 7.1. Velocity (left) and pressure (right) fields for the smooth Stokes.

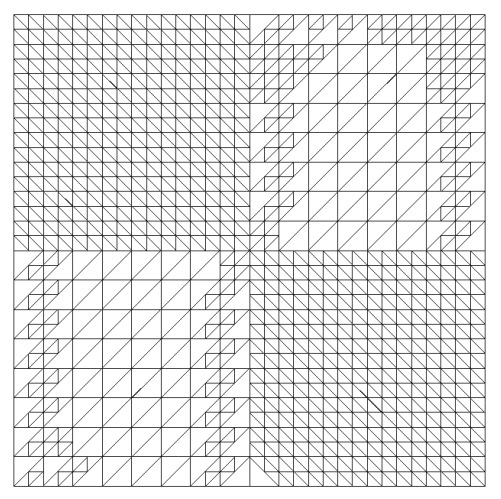

FiG. 7.2. Finite element mesh after several refinement steps for the smooth Stokes problem

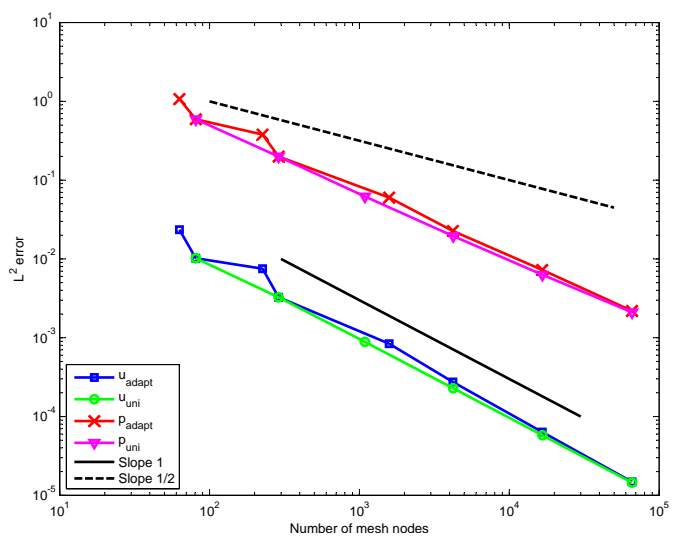

FIG. 7.3. Velocity and pressure convergence plots for the smooth Stokes problem.

7.2. Singular solution problem. In this numerical example we consider an L-shaped domain:

$$
\Omega:=((-1,1) \times(-1,1)) \backslash([0,1] \times[-1,0]) .
$$



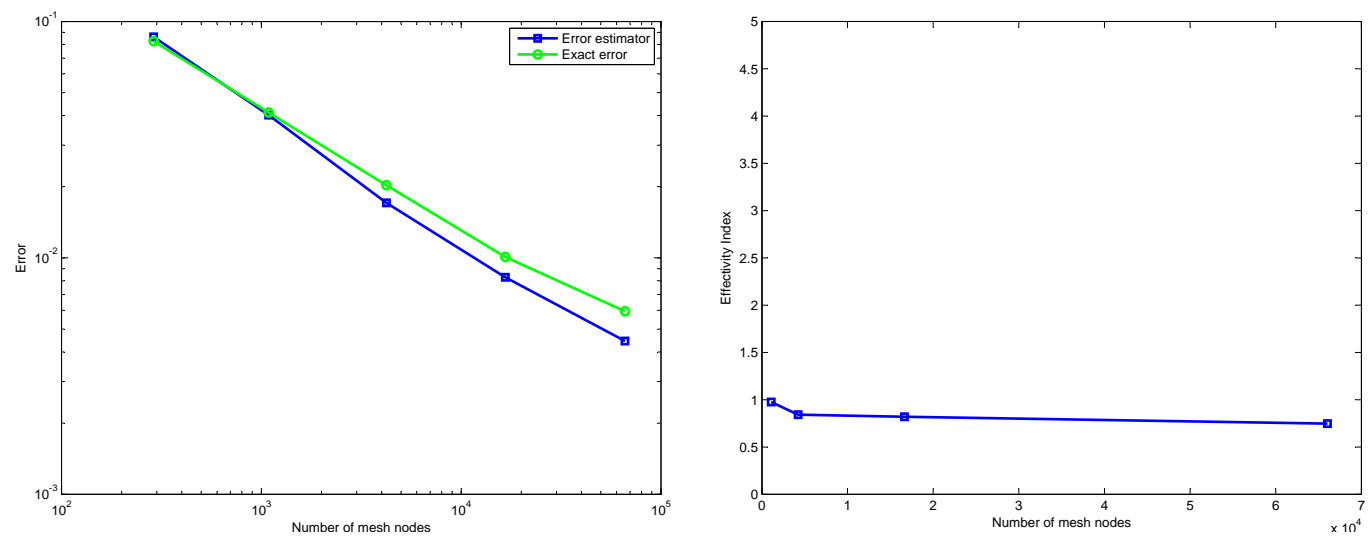

FIG. 7.4. Comparison between the estimated error and the actual error in the cdG energy norm 4.21) (left) and effectivity index (right) for the smooth solution Stokes problem.

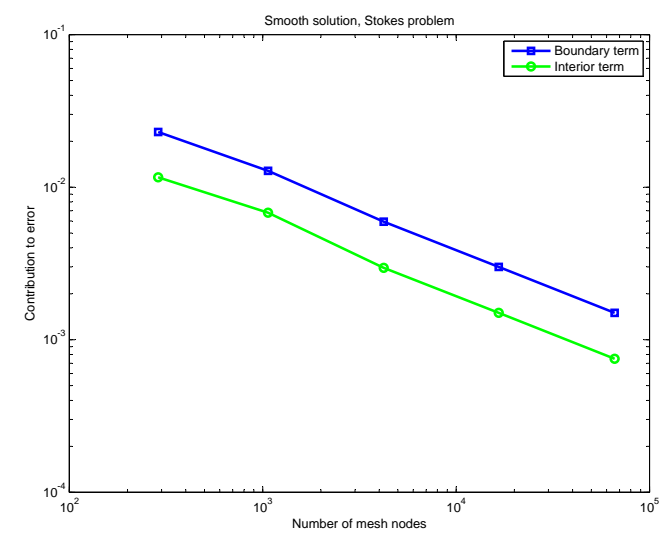

FIG. 7.5. Comparison between the boundary and interior terms contribution to the error estimator for the smooth Stokes problem.

In this domain, we consider a divergence-free analytical solution of the stationary Stokes problem (see [20]):

$$
\boldsymbol{u}(r, \phi)=r^{\alpha}\left[\begin{array}{c}
\cos (\phi) \psi^{\prime}(\phi)+(1+\alpha) \sin (\phi) \psi(\phi) \\
\sin (\phi) \psi^{\prime}(\phi)-(1+\alpha) \sin (\phi) \psi(\phi)
\end{array}\right], \quad p(r, \phi)=-r^{\alpha-1} \frac{(1+\alpha)^{2} \psi^{\prime}(\phi)+\psi^{\prime \prime \prime}(\phi)}{1-\alpha},
$$

with

$$
\psi(\phi)=\frac{\sin ((1+\alpha) \phi) \cos (\alpha \omega)}{1+\alpha}-\cos ((1+\alpha) \phi)+\frac{\sin ((\alpha-1) \phi) \cos (\alpha \omega)}{1-\alpha}+\cos ((\alpha-1) \phi) .
$$

$\omega$ and $\alpha$ are taken as $\omega=3 \pi / 2$ and $\alpha \approx 0.5444837$, which is an approximation to the root of the non-linear equation:

$$
\frac{\sin ^{2}(\alpha \omega)-\alpha^{2} \sin ^{2}(w)}{\alpha^{2}}=0 .
$$

Fig.7.7 shows the velocity and pressure fields obtained from the numerical simulations. In the reentrant corner, a pressure singularity can be observed. As in the previous numerical examples, we depart from a 6 linear element triangular FE mesh and we let Algorithm 2 refine until a certain element error is reached for each element. As expected from the pressure field pattern, a strong refinement appears in the vicinity of the corner, which allows the FE solution to better capture the sharp pressure gradients 


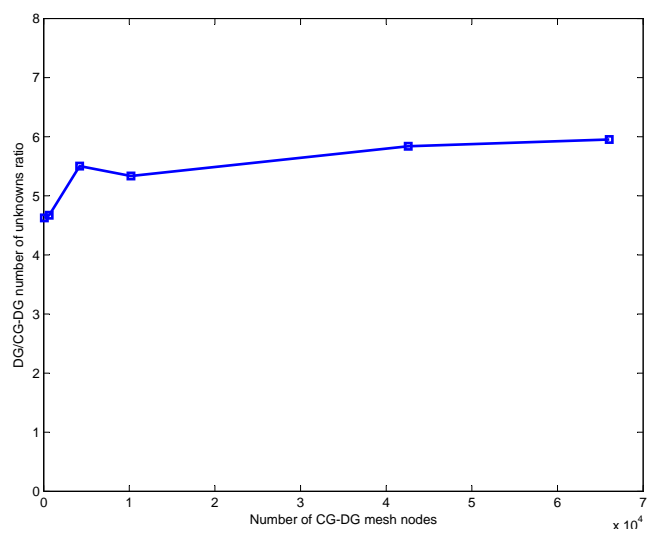

FIG. 7.6. Ratio between the number of unknowns of a pure $d G$ formulation and the proposed $c G$-dG formulation for the smooth Stokes problem.

(Fig.7.8). If we study the velocity and pressure convergence plots (Fig.7.9), we can observe that an optimal convergence rate is obtained for the velocity, and superconvergence is observed for the pressure values when our adaptive refinement strategy is used. On the other hand, uniform refinement performs poorly because the global error is governed by the error in the vicinity of the pressure singularity. Again, we study the performance of the error estimator in the adaptive refinement procedure. Fig. [7.10 shows the estimated error evaluated versus the exact error and the effectivity index. It can be observed that the convergence rate is approximately the same for the error estimator and the exact error. The effectivity index shows the reliability and efficiency of the estimator for singular solutions, around 1.5. Fig.7.11 shows the ratio between $h_{K} \sum_{K \in \mathcal{T}_{h}} \int_{\partial K \backslash \Gamma}\left|\llbracket \boldsymbol{\sigma}_{h} \rrbracket\right|^{2} \mathrm{~d} \boldsymbol{x}$ and $\left\|\boldsymbol{\sigma}_{h}-\pi_{h}^{*}\left(\boldsymbol{\sigma}_{h}\right)\right\|^{2}$. The figure illustrates the equivalence between both terms, which converge at the same rate. A comparison between the number of unknowns of the proposed cdG formulation and a pure $d G$ formulation for the same FE mesh is presented in Fig.7.12 This ratio is around 6 for this bidimensional problem.
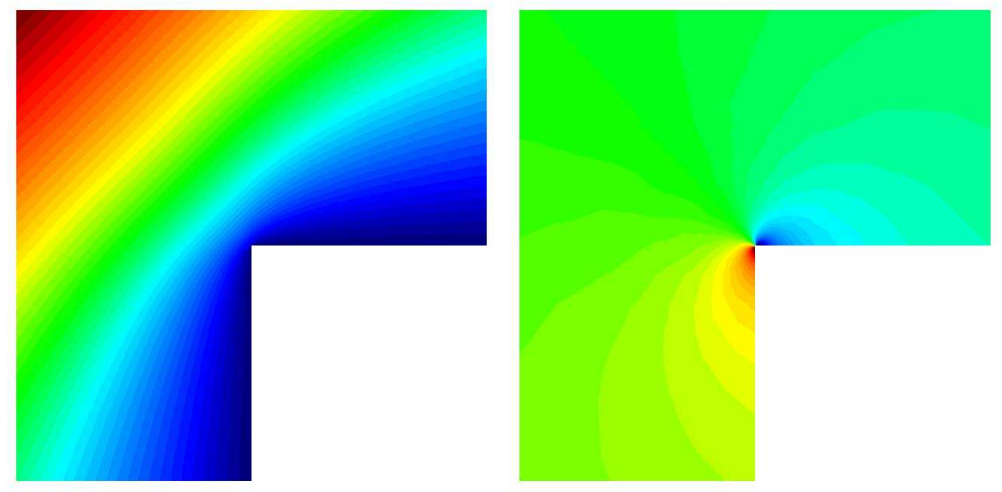

FIG. 7.7. Velocity (left) and pressure (right) fields for the singular solution Stokes problem.

7.3. Transient incompressible Navier-Stokes equations. In this numerical example we test the developed adaptive refinement strategy for the transient incompressible Navier-Stokes equations. We solve the incompressible flow around a cylinder at $R e=100$. The computational domain consists of a $16 \times 8$ rectangle with a unit-diameter cylinder centered at $(4,4)$. The horizontal inflow velocity is set to 100 at $x=0$. Slip boundary conditions which allow the flow to move in the direction parallel to the walls are set at $y=0$ and $y=8$, and velocity is set to $\mathbf{0}$ at the cylinder surface. The viscosity has been set to $\nu=1$, which yields a Reynolds number $\mathrm{Re}=100$ based on the diameter of the cylinder and the inflow velocity. A backward Euler scheme has been used for the time integration with time step $\delta t=2 \cdot 10^{-3}$. 

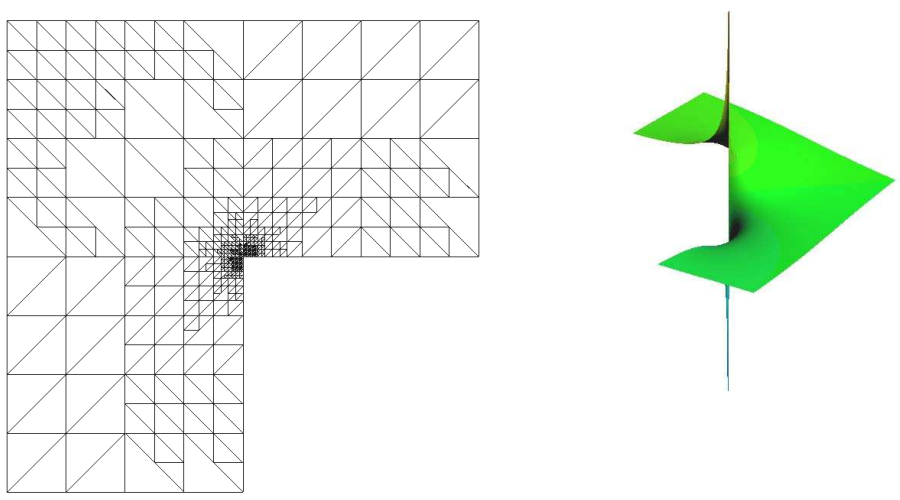

FIG. 7.8. Finite element mesh after several refinement steps (left) and pressure singularity in the corner of the domain (right) for the singular solution Stokes problem

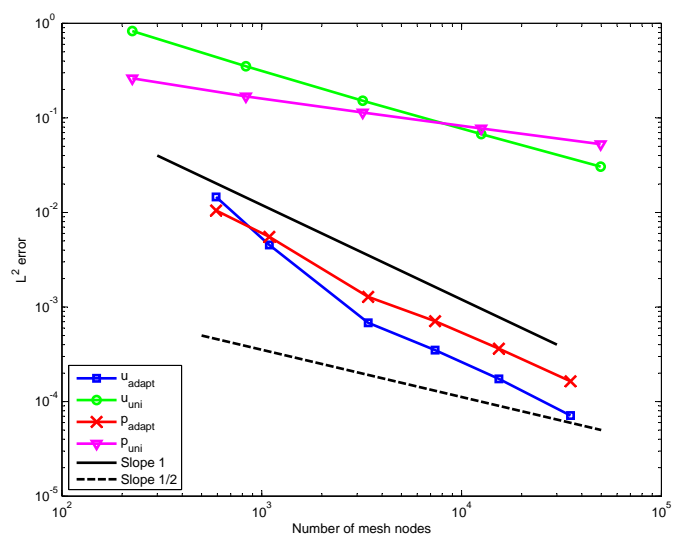

FiG. 7.9. Velocity and pressure convergence plots for the singular solution Stokes problem.

For the adaptive refinement strategy, we depart from a 7,294 linear triangle FE mesh. Contrary to the previous examples, here we use Algorithm 3, i.e. we fix an objective number of elements in the FE mesh, instead of fixing a local elemental error. The adaptive strategy refines the elements with the largest local errors and unrefines the elements with the smallest local errors so that the element errors are approximately uniform and the number of elements approaches the objective number of elements. This strategy results in an adaptive mesh which evolves in time with the FE solution, following the vortexes which appear behind the cylinder. The objective number of elements is set to 20,000 in this numerical experiment. Fig. 7.13 shows the original FE mesh and the adaptively refined meshes at several time steps of the simulation. The adaptive algorithm strongly refines the boundary layer region close to the cylinder. In this area the gradients are sharper and a finer mesh is required in order to accurately represent the solution. This refinement remains constant in time. On the other hand, the algorithm also refines in the domain regions in which the vortexes appearing at the cylinder wake produce large velocity gradients. Since the vortexes move in time, so does the adaptive mesh, as it is illustrated in Fig.7.13, After the flow is fully developed, the actual number of FEs which is obtained from the adaptive refinement ranges between 18,400 and 18,700, depending on the time step.

8. Conclusions. In this work, we have proposed an equal order cdG FE formulation for adaptive simulations of incompressible flows. The reason to use a hybrid cdG approach is motivated by the fact that $\mathrm{cG}$ methods are less CPU intensive for a given mesh whereas with dG methods we can naturally handle non-conforming meshes, which appear when cheap and simple isotropic local refinement is used; incompressible flows usually lead to solutions with sharp internal gradients and boundary layers, and adaptive refinement is required. In both cases, equal order interpolation is an optimal choice in the trade-off between accuracy and efficiency. 

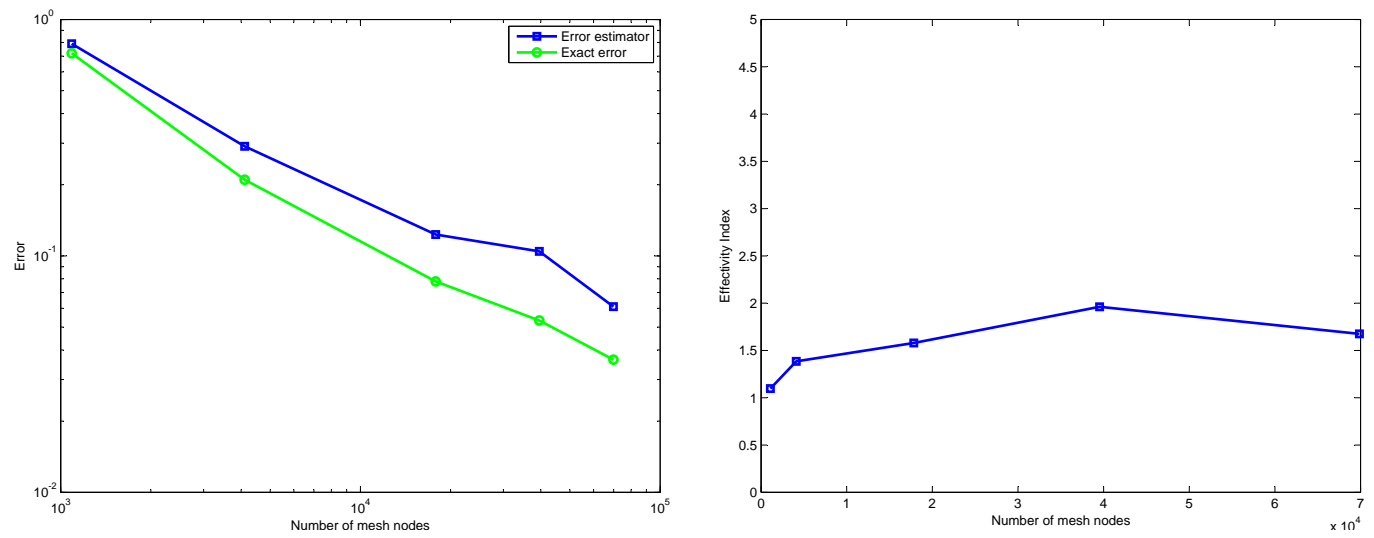

FIG. 7.10. Comparison between the estimated error and the actual error in the cdG energy norm 4.21) (left) and effectivity index (right) for the singular solution Stokes problem.

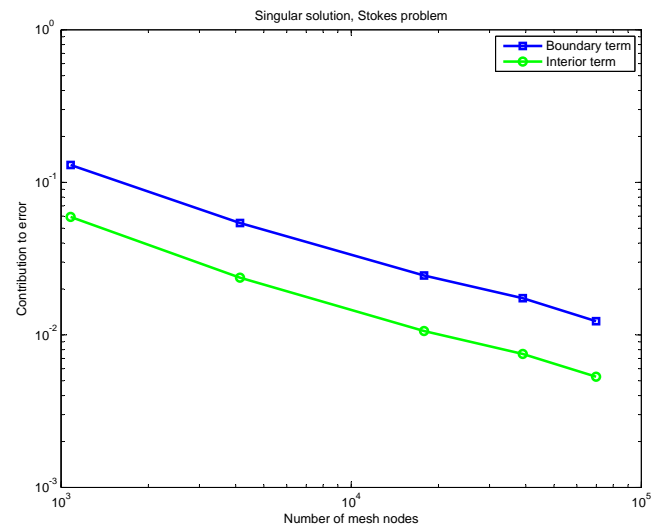

FIG. 7.11. Comparison between the boundary and interior terms contribution to the error estimator for the singular solution Stokes problem.

The simulation of incompressible flows via equal-order formulations requires to introduce stabilization terms, both in the $\mathrm{cG}$ and $\mathrm{dG}$ case. In both cases, pressure stability relies on the stabilization terms, via a weakened inf-sup condition. Convection stabilization is also required at moderate to high Reynolds numbers. In the cG case, this stability is attained by SUPG-type formulations, whereas proper flux definitions are needed in the $\mathrm{dG}$ case. The use of cdG FE spaces is not straightforward, because both the $\mathrm{cG}$ and $\mathrm{dG}$ formulations miss terms that are needed in the hybrid case. In this work, we design a cdG formulation that combines the VMS cG stabilization in 26] and the equal-order $\mathrm{dG}$ formulation in [15. with classical upwind fluxes for the convective term [13, 38. The resulting method is proved to be stable and convergent for the Stokes system.

Further, we have combined this cdG formulation with a suitable adaptive refinement strategy, in order to produce numerical experiments on adapted meshes. On adapted (non-conforming) FE meshes, the cdG method exhibits the optimal convergence properties obtained form the numerical analysis while keeping a low $\mathrm{CPU}$ cost compared to the $\mathrm{dG}$ case. The error estimators have shown good performance for smooth and singular solutions of the Stokes problem, as well as for the transient incompressible Navier-Stokes equations.

Future work will focus on the extension of the cdG approach to $h p$-adaptivity and the numerical analysis of the error estimates in this setting. 


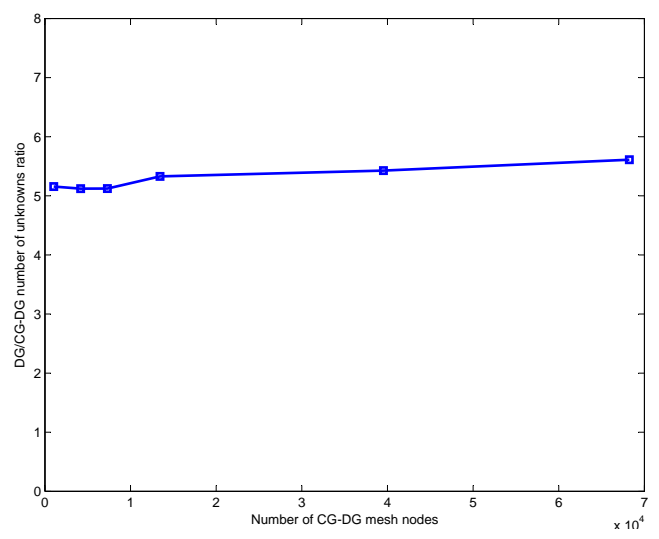

FIG. 7.12. Ratio between the number of unknowns of a pure $d G$ formulation and the proposed cdG formulation for the singular solution Stokes problem.

[1] B. Aksoylu, S. Bond, and M. Holst. An odyssey into local refinement and multilevel preconditioning III: implementation and numerical experiments. SIAM Journal on Scientific Computing, 25(2):478-498, 2003.

[2] D. N. Arnold, F. Brezzi, B. Cockburn, and L. D. Marini. Unified analysis of discontinuous Galerkin methods for elliptic problems. SIAM Journal on Numerical Analysis, 39(5):1749-1779, 2002.

[3] S. Badia. On stabilized finite element methods based on the Scott-Zhang projector. Circumventing the inf-sup condition for the Stokes problem. Computer Methods in Applied Mechanics and Engineering, 247-248:65-72, 2012.

[4] S. Badia and R. Codina. Unified stabilized finite element formulations for the Stokes and the Darcy problems. SIAM Journal on Numerical Analysis, 47(3):1971-2000, 2009.

[5] S. Badia and R. Codina. A combined nodal continuous-discontinuous finite element formulation for the Maxwell problem. Applied Mathematics and Computation, 218(8):4276-4294, 2011.

[6] S. Badia and R. Codina. On the design of discontinuous Galerkin methods for elliptic problems based on hybrid formulations. Submitted, 2012.

[7] J. Baiges, R. Codina, F. Henke, S. Shahmiri, and W.A. Wall. A symmetric method for weakly imposing Dirichlet boundary conditions in embedded finite element meshes. International Journal for Numerical Methods in Engineering, 90:636-658, 2012.

[8] R. Becker and M. Braack. A finite element pressure gradient stabilization for the Stokes equations based on local projections. Calcolo, 38(4):173-199, 2001.

[9] S. Berrone, V. Garbero, and M. Marro. Numerical simulation of low-Reynolds number flows past rectangular cylinders based on adaptive finite element and finite volume methods. Computers E Fluids, 40(1):92-112, 2011.

[10] S. Berrone and M. Marro. Space-time adaptive simulations for unsteady Navier-Stokes problems. Computers 83 Fluids, 38(6):1132-1144, 2009.

[11] S. C. Brenner and R. Scott. The Mathematical Theory of Finite Element Methods. Springer, 2010.

[12] F. Brezzi and M. Fortin. Mixed and Hybrid Finite Element Methods. Springer-Verlag, 1991.

[13] F. Brezzi, L. D. Marini, and E. Süli. Discontinuous Galerkin methods for first-order hyperbolic problems. Mathematical Models and Methods in Applied Sciences (M3AS), 14(12):1893-1903, 2004.

[14] E. Burman, M. A. Fernández, and P. Hansbo. Continuous interior penalty finite element method for Oseen's equations. SIAM Journal on Numerical Analysis, 44(3):1248-1274, 2006.

[15] B. Cockburn, G. Kanschat, and D. Schötzau. An equal-order DG method for the incompressible Navier-Stokes equations. Journal of Scientific Computing, 40(1):188-210, 2009.

[16] B. Cockburn, G. Kanschat, D. Schotzau, and C. Schwab. Local discontinuous Galerkin methods for the stokes system. SIAM Journal on Numerical Analysis, 40(1):319, 2002.

[17] R. Codina. Stabilized finite element approximation of transient incompressible flows using orthogonal subscales. Computer Methods in Applied Mechanics and Engineering, 191(39-40):4295-4321, 2002.

[18] R. Codina and J. Blasco. A finite element formulation for the Stokes problem allowing equal velocity-pressure interpolation. Computer Methods in Applied Mechanics and Engineering, 143(3-4):373-391, 1997.

[19] R. Codina, J. Principe, O. Guasch, and S. Badia. Time dependent subscales in the stabilized finite element approximation of incompressible flow problems. Computer Methods in Applied Mechanics and Engineering, 196(2124):2413-2430, 2007.

[20] M. Dauge. Stationary Stokes and Navier-Stokes systems on two- or three-dimensional domains with corners. Part I: Linearized equations. SIAM Journal on Mathematical Analysis, 20(1):74-97, 1989.

[21] A. Ern and J.-L. Guermond. Theory and Practice of Finite Elements. Springer, 2004.

[22] P. Houston, D. Schötzau, and T. P. Wihler. Energy norm A Posteriori error estimation for mixed discontinuous Galerkin approximations of the Stokes problem. Journal of Scientific Computing, 22-23(1):347-370, 2005.

[23] P. Houston, D. Schötzau, and T. P. Wihler. An $h p$-adaptive mixed discontinuous Galerkin FEM for nearly incompressible linear elasticity. Computer Methods in Applied Mechanics and Engineering, 195(25-28):3224-3246, 2006. 

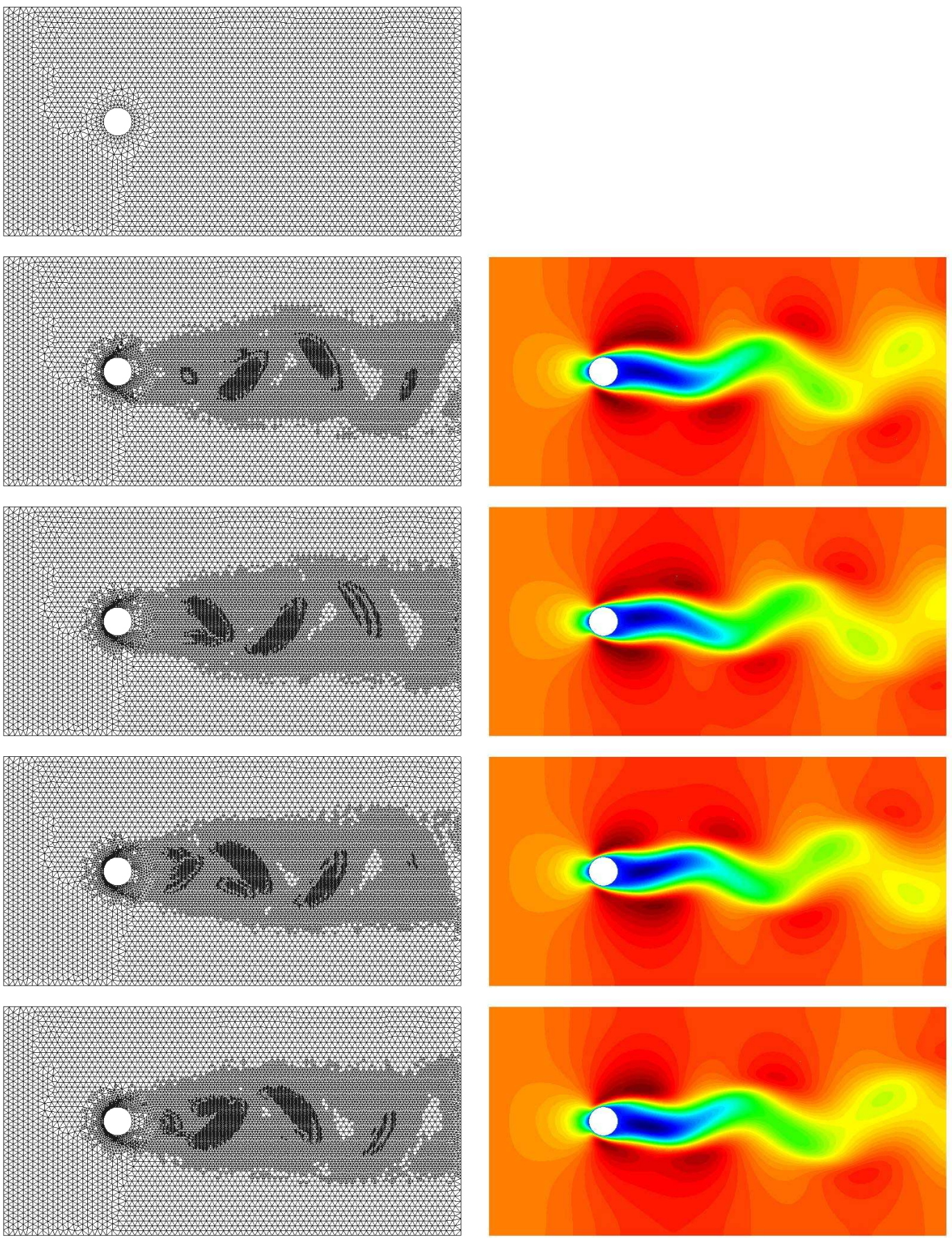

FIG. 7.13. Adaptive FE meshes and the corresponding velocity fields at several time steps of the simulation. Incompressible flow around a cylinder.

[24] P. Houston, D. Schötzau, and T. P. Wihler. Energy norm a posteriori error estimation of $h p$-adaptive discontinuous Galerkin methods for elliptic problems. Mathematical Models and Methods in Applied Sciences (M3AS), 17(1):33-62, 2007.

[25] T.J.R. Hughes. Multiscale phenomena: Green's functions, the Dirichlet-to-Neumann formulation, subgrid scale models, bubbles and the origins of stabilized methods. Computer Methods in Applied Mechanics and Engineering, 127(1-4):387-401, 1995.

[26] T.J.R. Hughes, G. R. Feijóo, L. Mazzei, and J.-B. Quincy. The variational multiscale method-a paradigm for computational mechanics. Computer Methods in Applied Mechanics and Engineering, 166(1-2):3-24, 1998. 
[27] O. A. Karakashian and F. Pascal. A posteriori error estimates for a discontinuous Galerkin approximation of secondorder elliptic problems. SIAM Journal on Numerical Analysis, 41(6):2374-2399, 2003.

[28] El Alaoui L. and A. Ern. Residual and hierarchical a posteriori error estimates for nonconforming mixed finite element methods. ESAIM: Mathematical Modelling and Numerical Analysis, 38(6):903-929, 2004.

[29] G. Matthies, P. Skrzypacz, and L. Tobiska. A unified convergence analysis for local projection stabilisations applied to the Oseen problem. ESAIM: Mathematical Modelling and Numerical Analysis, 41(4):713-742, 2007.

[30] J. Nitsche. Über ein Variationsprinzip zur Lösung von Dirichlet-Problemen bei Verwendung von Teilräumen, die keinen Randbedingungen unterworfen sind. Abhandlungen aus dem Mathematischen Seminar der Universitt Hamburg, 36(1):9-15, 1971.

[31] S. Norburn and D. Silvester. Stabilised vs. stable mixed methods for incompressible flow. Computer Methods in Applied Mechanics and Engineering, 166(1-2):131-141, 1998

[32] I. Perugia and D. Schötzau. An $h p$-Analysis of the local discontinuous Galerkin method for diffusion problems. Journal of Scientific Computing, 17(1):561-571, 2002.

[33] W. Rachowicz and L. Demkowicz. An $h p$-adaptive finite element method for electromagnetics-part II: a 3D implementation. International Journal for Numerical Methods in Engineering, 53(1):147-180, 2001.

[34] D. Schötzau, C. Schwab, and A. Toselli. Mixed hp-DGFEM for incompressible flows. SIAM Journal on Numerical Analysis, 40(6):2171-2194, 2003.

[35] L. R. Scott and S. Zhang. Finite element interpolation of nonsmooth functions satisfying boundary conditions. Mathematics of Computation, 54(190):483-493, 1990.

[36] P. Solín, K. Segeth, and I. Dolezel. Higher-Order Finite Element Methods. Chapman \& Hall/CRC, 2004.

[37] R. Temam. Navier-Stokes Equations: Theory and Numerical Analysis. American Mathematical Society, 2000.

[38] L. Zhu and D. Schötzau. A robust a posteriori error estimate for $h p$-adaptive DG methods for convection-diffusion equations. IMA Journal of Numerical Analysis, 31(3):971 -1005, 2011.

[39] O.C. Zienkiewicz and J.Z. Zhu. The superconvergent patch recovery and a posteriori error estimates. Part 1: The recovery technique. International Journal for Numerical Methods in Engineering, 33:1331-1364, 1992.

[40] O.C. Zienkiewicz and J.Z. Zhu. The superconvergent patch recovery and a posteriori error estimates. Part 2: Error estimates and adaptivity. International Journal for Numerical Methods in Engineering, 33:1365-1382, 1992. 